\title{
FRANCISCO GUERRERO HÉRITIER DE LA TRADITION FRANCO-FLAMANDE: ONZE PIÈCES BASÉES SUR UN OSTINATO
}

Olivier TRACHIER

\begin{abstract}
In his religious works, Francisco Guerrero several times uses a type of contrapuntal composition rather unusual in his period: writing on cantus firmus ostinato. For German musicologists, it represents one of the last forms of the medieval isorythmic motet. The architectural study, especially elaborate, is regulated by a real musical arithmetic scheme issued from ars musica mathematica. Therefore, Guerrero dignifies his predecessors tradition illustrated by the Josquin des Prés's Miserere for 5 voices. He also succeeds his master Cristobal de Morales, appearing to make use of some of the former's models. Nevertheless he knows how to compose in an up-to-date manner and give oratorial meaning and efficiency to the ostinato, as the greatest rhetoricians. This peculiarity of this musical personality harmoniously combines with its innovating side -that of villancicos and polychorals motets-in order to give evidence of his universal genius.
\end{abstract}

\section{Résumé}

Dans son œuvre religieuse, Francisco Guerrero exploite à plusieurs reprises un type de composition contrapuntique relativement peu répandu à son époque: l'écriture sur cantus firmus ostinato. Pour les musicologues germaniques, ceci représente une des formes ultimes du motet isorythmique médiéval. Le travail architectural, particulièrement soigné, $\mathrm{y}$ est ordonné par une véritable arithmétique musicale, issue de l'ars musica mathematica. Guerrero honore ainsi la tradition de ses prédécesseurs, illustrée par le Miserere à 5 voix de Josquin des Prés. Il suit également son maître Cristobal de Morales en lui empruntant, semble-t-il, quelques modèles. Mais son écriture sait se mettre au goût du jour, et confère à l'ostinato un sens et une efficacité oratoires dignes d'un grand rhétoricien. Cet aspect de sa personnalité musicale s'unit harmonieusement à son côté novateur-celui des villancicos et des motets polychorauxpour témoigner de son génie universel.

\section{Introduction}

L'œuvre de Francisco Guerrero présente un certain nombre d'aspects typiquement ibériques, comme les villancicos, cançiones et villanescas. Un style plus international paraît caractériser beaucoup de pièces religieuses. Ainsi, le lien du compositeur avec la tradition franco-flamande s'affirme avec netteté dans quelques motets et extraits de messe bâtis autour d'un ostinato. 
Les huit volumes publiés à ce jour par le Consejo Superior de Investigaciones Científicas ${ }^{1}$ permettent en effet de sélectionner onze pièces où l'une des parties vocales répète un même motif du début jusqu'à la fin. Il s’agit, par ordre chronologique ${ }^{2}$, de:

Veni Domine (motet-1555); vol. 6, $\mathrm{n}^{\circ} \mathrm{XXXI}$ pp. 106-110;

Missa In te Domine: Sanctus (1566); vol. 7, $\mathrm{n}^{\circ} \mathrm{XI}$ pp. 171-172;

Missa Sancta et immaculata: Sanctus, Benedictus (1566); vol. 7, $\mathrm{n}^{\circ} \mathrm{X}$ pp. 130-131 et 136137;

Missa Congratulamini: Agnus I (1566); vol. 7, $\mathrm{n}^{\circ}$ XII pp. 218-220;

Missa Beata mater: Hosanna (1566); vol. 7, n IX p. 96;

Surge propera (motet-1570); vol. 3, $\mathrm{n}^{\circ} \mathrm{XX}$ pp. 116-125;

Missa Ecce sacerdos: Kyrie II (1582); vol. $4 \mathrm{n}^{\circ}$ II pp. 40-42;

Missa della Batalla: Agnus I (1582); vol. 4 n III pp. 106-109;

Missa Simile est: Agnus I (1582); vol. 5, n VI pp. 88-89;

Missa seculorum Amen: Agnus I (1597); vol. 9, $\mathrm{n}^{\circ}$ XVIII pp. 133-135.

La technique employée se réfère à des modèles antérieurs, dont les plus célèbres, comme le grand Miserere de Josquin des Prés, ont été imités plusieurs fois par de nombreux compositeurs tout au long du XVIe s. A ce propos, il n'est pas inutile de rappeler l'historique de ce procédé de composition et de montrer la place importante qu'occupe la production guerrérienne en son sein. C'est l'objet du premier chapitre de cet article. A côté de cet aspect-là, il convient de s'attacher à l'analyse des musiques. Différentes études ayant récemment établi plusieurs typologies d'ostinato ${ }^{3}$, il paraît souhaitable de les mettre en regard et de tester leur efficacité, par exemple sur les onze pièces du Maître sévillan.

L'analyse purement technique peut alors être complétée avantageusement par les éléments issus d'autres aspects de la culture ancienne, comme la rhétorique, les mathématiques (et même l'héraldique, comme on le verra plus loin). Ces sciences -ou plutôt ces arts- entretiennent à la Renaissance des rapports étroits avec l'ars musica et permettent de mieux saisir la réalité complexe de l'œuvre dans son contexte historique ${ }^{4}$. La description précise de chaque pièce de Francisco Guerrero suit donc ici plusieurs pistes d'analyse, unies par leur caractère émique. On tente ainsi une interprétation plurielle qui touche aux domaines de la symbolique et de la sémantique. L'originalité et la grandeur du Maestro s'illustrent alors avec force.

1. Dans la série Monumentos de la Música española, sous la direction de V. García et J. M. Llorens Cisteró, Barcelona 1955-1997.

2. Il s'agit de l'ordre chronologique de la publication au XVIe s. On précise, pour chaque pièce, le numéro du volume, le numéro d'ordre et la pagination dans l'édition monumentale. La description précise de l'ostinato est faite au chapitre III.

3. Les références sont données plus bas, au chapitre II.

4. Voir à ce propos Gallus Dressler: Praecepta Musicae Poëticae, présentation et traduction par Olivier Trachier, Paris, Klincksieck-CESR, 1999 (sous presse). 


\section{L'ostinato dans la musique religieuse aux XVe et XVIe siècles}

Aborder l'historique d'un mode d'écriture si particulier oblige à prendre en compte plus généralement l'évolution de la composition et celle des genres principaux. L'objet de cette étude permettant de laisser de côté le répertoire profane, où l'ostinato est nettement moins représenté c'est le motet et la messe qui requièrent toute notre attention.

1. Le motet et la messe. Entre le début et la fin du XVe s., le traitement du motet se transforme profondément. Comme le montre très clairement Ludwig Finscher dans son étude Arten der Motette in 15. und 16. Jahrhundert ${ }^{6}$, l'un des axes essentiels de ce processus concerne le passage du motet isorythmique au Tenormotette, motet bâti autour d'un ténor. La pluritextualité tend également à se réduire. Il est clair qu'au tournant du XVIe s., ce genre musical s'oriente vers l'union syntaxique du texte (unique) et de la musique plutôt que vers des constructions arithmétiques savantes ${ }^{7}$. La grande majorité des pièces travaille à l'émergence d'un style nommé au XXe s. "style imitatif syntaxique" par le musicologue belge Charles van den Borren $^{8}$, et qui trouve ses lettres de noblesse au temps de Lassus et de Palestrina.

De même, le mode et l'ordre de composition des voix paraissent marqués par une diversité de plus en plus grande ${ }^{9}$. Par-delà le clivage entre isorythmie et préséance d'un ténor, on constate que la hiérarchie vocale tend à s'égaliser ${ }^{10}$.

Est-ce à dire que l'isorythmie disparaît totalement à la fin du XVe s. et que le ténor perd toute importance au temps de la Réforme? Bien au contraire, et dans ce domaine, la composition du motet sur ostinato apporte un démenti formel à ces affirmations. En effet, la musique y est construite autour d'un ténor, qui a souvent son propre texte littéraire ${ }^{11}$; sa disposition est très rigoureusement proportionnée: les interventions de l'ostinato et les pauses qui les séparent sont calibrées suivant un ou plusieurs étalons, généralement fixes à l'intérieur de la pièce ${ }^{12}$. On reconnaît là l'architecture vocale du motet isorythmique, avec deux différences importantes, toutefois: ce qui fait office de talea dans le cas présent ne souffre aucune variante mélodique, rythme et musique étant indéfectiblement liés, et ceci ne concerne qu'une seule voix.

Ce type de travail se retrouve dans la messe bâtie sur un cantus firmus. Certaines parties, généralement assez brèves, traitent une portion de la mélodie obligée sous forme d'ostinato; ainsi, le premier ou le second Kyrie, le Sanctus, le Benedictus ou l'Agnus peuvent être construits

5. Guerrero ne s'en sert pas, au sens strict, dans ses cançiones.

6. Neues Handbuch der Musikwissenschaft, Band 3,2: Die Musik des 15. und 16. Jahrhunderts, édité par Carl Dahlhaus et Ludwig Finscher, Laaber, Laaber-Verlag, 1990, pp. 277-324.

7. Voir à ce propos Philippe Vendrix: La Musique à la Renaissance, Paris, PUF "Que sais-je", 1999.

8. Quelques réflexions sur le style imitatif syntaxique, Revue belge de Musicologie I (1946-47), pp. 14-20.

9. Voir à ce propos Jessie-Ann Owens: Composers at Work, New York, Oxford University Press, 1997.

10. Gallus Dressler (op. cit., chapitre VI) relativise le nom-même de ténor, qui représente, pour lui, la voix "choisie pour composer le thème" de tout chant polyphonique, "qu'il s'agisse du discantus, du bassus, ou de toute autre partie".

11. Dans ce cas, la pluritextualité ne désigne qu'une simple dualité. Par ailleurs, le terme de "ténor" doit être pris au sens large, tel que le conçoit Gallus Dressler (cf. note 10): l'ostinato est appliqué librement à toute partie vocale. ternaire, etc.

12. Sauf lorsqu'il y a un travail interne de mise en proportion: ostinato repris par diminution ou lu en mesure 
de cette façon. L'extension du procédé à une section entière de la messe comme le Gloria ou le Credo (écrits tout d'une pièce) est plus rare ${ }^{13}$. Parfois, ce sont des fragments de messe-parodie qui se présentent ainsi ${ }^{14}$.

2. Listes d'œuvres. Dans la musique sacrée en général, les musicologues notent l'emploi d'un ostinato dans certaines œuvres de G. Dufay comme le Gloria ad modum tubae ou le Gloria de quaremiaulx $x^{15}$.

De même, les motets In hydraulis et Anthoni usque limina (avant 1467) d'Antoine Busnois ${ }^{16}$ se classent parmi les plus anciennes manifestations de cette technique. La voce ostinata est parfois d'une extrême simplicité, comme dans les œuvres écrites à l'imitation des cloches ou des trompettes (ad modum campanarum/tubarum); ainsi, le second motet de Busnois cité cidessus ne répète qu'une seule note, qui était peut-être jouée par une cloche, selon Gustave Reese $^{17}$. Dans d'autres cas, il s'agit d'une période entière (Dufay, Gloria de quaremiaulx).

L'histoire n'accorde pas une place éminente à ces pièces-là. Il faut attendre Josquin des Prés pour voir couronner quelques chefs-d'œuvre illustrant cette technique. Les messes et les motets où le compositeur utilise l'ostinato comptent parmi les plus célèbres; ils ont un succès durable et suscitent bon nombre d'imitations tout au long du XVIe s. On cite volontiers les motets:

Illibata Dei virgo, Smijers $n^{\circ} 27$; ostinato la mi la au ténor;

Miserere mei Deus, Sm. ${ }^{\circ} 37$; ost. (même texte) au ténor 2;

Salve regina (5 voix), Sm. $\mathrm{n}^{\circ} 48$; ost. salve au quintus;

Domine Deus noster, Sm. $\mathrm{n}^{\circ} 89$. ost. (même texte) au ténor 2;

et les messes ${ }^{18}$ :

La sol fa re $m i, \mathrm{Sm} . \mathrm{n}^{\circ} \mathrm{II}$; répétitions diverses de ces notes, à différentes voix;

Gaudeamus, Sm. n' III; ost. : incipit de l'introït du même nom, en particulier dans le Sanctus, l'Hosanna, l'Agnus III;

13. Voir Josquin, Missa Hercules dux Ferrariae, Gloria et Credo.

14. Voir Guerrero, Missa Sancta et Immaculata, Benedictus.

15. Il s'agit des pièces $\mathrm{n}^{\circ} 22$ et 23 éditées par Heinrich Besseler dans le vol. 4 des Opera omnia. Elles sont citées

par:

-Ernst Apfel, Untersuchung zur Entstehung und Frühgeschichte des Ostinato in der komponierten Mehrstimmigkeit, Saarbrucken, chez l'auteur, 1976, pp. 11-12,

-Willem Elders, Zur Auffuhrungspraxis der altniederländischen Musik, in: Mélanges, R. B. Lenaerts ed., Leuven, 1969, pp. 91-93. col. 1237.

-(Ière pièce seulement) Georg Reichert et Michaël von Troschke, Ostinato, MGG-VII, Barenreiter-Metzler, 1997,

-(Ière pièce seulement) Frédéric Billiet, Le cantus firmus enigmatique dans le répertoire polyphonique dit francoflamand, in: Itinéraires du cantus firmus III, Edith Weber ed., Presses de l'Université Paris-Sorbonne, 1999, p. 75.

16. Le premier motet se trouve dans DTÖ VII, vol. 14/15, p. 105, et le second dans Het Anthonius-Motet van Anthonius Busnois, C. L. Walther Boer ed., Amsterdam 1940.

17. Music in the Renaissance, London, 1964, p. 109.

18. La liste n'est pas exhaustive. Mais les quatre messes citées contiennent, proportionnellement, le plus grand nombre d'ostinatos. Le lecteur pourra également consulter certaines parties de messes comme le Benedictus de la Missa Faisant regretz ou le Kyrie II de la Missa Ave maris stella. 
Hercules dux Ferrariae, Sm. $\mathrm{n}^{\circ}$ VII; ost. solmistique (voir chapitre II), presque toujours au ténor;

L'ami Baudichon, $\mathrm{Sm} . \mathrm{n}^{\circ} \mathrm{IX}$; le ténor répète souvent les notes mi ré do, avec quelques variantes.

Dans ces œuvres, Josquin présente l'ostinato de différentes façons ${ }^{19}$. On les étudie au chapitre suivant. Signalons néanmoins que ce sont les pièces où cette technique est exploitée avec le plus de simplicité -répétition identique dans la même voix- qui ont eu la plus grande postérité.

Ainsi, le ténor miserere mei Deus se retrouve, plus ou moins modifié rythmiquement, dans les motets suivants ${ }^{20}$ :

Misereatur mei, motet de Jean Richafort (1532); ostinato à l'altus;

Peccantem me quotidie, motet de Jachet Berchem (1542); ostinato à la sexta vox;

in: CMM III (A. Willaert Opera omnia), vol. 4, p. 109;

Mater digna Dei, motet de Jacobus Vaet (1562); ost. au ténor dans la seconde partie;

Sämtliche Werke II, DTÖ, Band 100, motet $n^{\circ} 31$;

Tribularer, motet de Palestrina (1572); ost. miserere mei Deus au sextus; Sämtliche

Werke, ed. F. X. Haberl, vol. 2, n 18.

Quant à la messe Hercules, elle sert de modèle à la Missa Vivat felix Hercules de Ciprien de Rore $^{21}$, à la Missa Philippus rex Hispaniae de Bartholomeo de Escobedo (1556), ainsi qu'à diverses pièces ${ }^{22}$. Différents compositeurs du XVIe s. écrivent également des messes sur un court motif, comme le la sol fa re mi de Josquin. Plusieurs parties de l'œuvre traitent alors la mélodie d'une façon répétitive. On ne dresse pas de liste correspondante, devant l'abondance de la matière et l'aspect parfois un peu trop libre de la répétition, qui n'entre pas bien dans la catégorie énoncée ci-dessus, marquée par une grande fixité.

D'une façon générale, il semble que le motet sur ostinato ${ }^{23}$ devienne, après Josquin et ses contemporains, une sorte de passage obligé dans toute carrière de compositeur, et ce jusqu'à la fin du XVle s. De telles pièces font un peu exception dans les productions individuelles, mais quelques maîtres semblent marquer une certaine prédilection pour ce type d'écriture. Il s'agit essentiellement de compositeurs vivant au milieu du XVle s. comme Clemens non Papa, A. Willaert ou C. de Morales, qui ont subi fortement l'influence de la génération josquinienne. A

19. On ne retient pas les messes qui ne présentent qu'un usage fugitif de la répétition ou redicta; voir chapitre II.

20. Voir à ce sujet W. Elders, Studien zur Symbolik in der Musik der alten Niederländern, Bilthoven, A. B. Creyghton, 1968, pp. 76-78. Ludwig Senfl signe un motet du même nom (cf. p. suiv.), avec un ostinato au ténor I; mais la mélodie est très différente.

21. Opera omnia CMM XIV, vol. 7, p. 32.

22. Voir W. Elders, op. cit. 1968, pp. 79-80.

23. Et la messe, dans une moindre mesure. Ceci ne concerne d'ailleurs qu'une de ses parties, dans la plupart des cas. Consulter la petite liste donnée pp. 57 et 58 . 
l'approche du siècle suivant, ce travail tombe un peu en désuétude. La pratique de l'ostinato ne disparaît pas, mais elle se transforme ${ }^{24}$ et recueille davantage de suffrages dans le répertoire profane (danses, musique instrumentale). La liste suivante, non exhaustive et limitée au XVIe s., s'attache à des pièces très représentatives et montre l'importance des Espagnols dans ce domaine, Morales en premier lieu. L'un des derniers représentants de cette école, Ferdinando de Las Infantas, illustre son attachement à la tradition franco-flamande en écrivant un bon nombre de motets sur ostinato ${ }^{25}$. C'est d'ailleurs l'avant-dernier nom de la série ${ }^{26}$.

Jean Richafort: $\quad$ Misereatur mei (voir ci-dessus);

Ludwig Senfl:

Miserere mei Deus; ost. au ténor I; Sämtliche Werke, ed. W.

Gerstenberg, vol. 3, p. 53;

Omnes gentes; ost. omnes gentes... au ténor I; ibid., p. 65;

Adrian Willaert: $\quad$ Peccavi; ost. tibi soli peccavi au quintus; CMM III, vol. 3, p. 61;

Victor io, salve; ost. Salve sfortiarum... au ténor; ibid., p. 90;

Ave Maria; ost. ave Maria au quintus; ibid., p. 114;

Cr. de Morales: $\quad$ Tu es Petrus; ost. tu es Petrus au cantus II;

Opera omnia vol. 2, ${ }^{\circ} \mathrm{XX}, \mathrm{p} .149$;

Andreas Christi famulus; ost. sancte Andrea ora pro nobis au cantus II; ibid., p. 157;

Exaltata est; ost. Virgo prudentissima au cantus II; ibid., p. 174;

Jubilate Deo; ost. gaudeamus, déjà traité par Josquin, au ténor I; ibid., p. 184;

Gaude et laetare; ost. magnificabo nomen tuum in aeternum à l'altus I; ibid., p. 192;

Veni Domine; ost. à l'altus II; vol. 5, p. 146;

Emendemus in melius; ost. memento homo... à l'altus II; vol. 8, p. 73;

Jachet Berchem: Peccantem me quotidie (cf. p. prec.);

Clemens non Papa: Fremuit spiritu Jesus; ost. Lazare veni foras au cantus II; CMM IV, vol. 14, p. 32;

Tota pulchra es; ost. sancta Margaretha ora pro nobis au quintus; CMM IV, vol. 16, p. 120;

Simon Moreau: $\quad$ Tu es Petrus; ost. tu es Petrus au quintus ${ }^{27}$, comme Morales; Frescobaldi.

24. Lire ainsi le Capriccio XI (1624) et le Ricercare con obligo di cantare la quinta parte (1635) pour orgue, de G.

25. Dans l'Espagne de Philippe II, une telle révérence peut passer, il est vrai, pour un certain rigorisme culturel, qui convient assez bien à la personnalité de Las Infantas. résumées.

26. Le classement suit la chronologie des compositeurs, pas celle des œuvres. Les références bibliographiques sont presse).

27. Publié dans Gallus Dressler: Practica Modorum Explicatio, ed. Olivier Trachier, Paris, RITM, 1999 (sous 
Fr. Guerrero: $\quad$ voir ci-dessus, page 55, et chapitre III;

Palestrina : $\quad$ Tribularer (cf. p. prec.);

Jacobus Vaet: $\quad$ Mater digna Dei (cf. p. prec.);

Roland de Lassus: Exsultet caelum; ost. quis audivit talia au ténor; Opera omnia, ed. F. X. Haberl, vol. 3, p. 144;

Fremuit spiritu Jesus; ost. Lazare veni foras au cantus II, comme Clemens non Papa; vol. 15, p. 23;

Homo cum in honore; ost. nosce teipsum à l'altus II; ibid., p. 90;

T. L. de Victoria: Salve regina (6 voix); ost. salve au cantus II, comme Josquin;

Opera omnia, vol. 2, p. 108;

Las Infantas: $\quad$ Missus est (6 voix, 1579); ost. ave Maria au sextus;

Veni Domine (6 v., 1579); ost. au sextus; pièce bâtie sur le même texte que le motet de Morales;

Laetetur omne saeculum (6 v., 1579); ost. au sextus;

Pater noster (6 v., 1579); ost. au sextus;

Jubilate Deo (6 v., 1579); ost. gaudeamus, déjà utilisé par Josquin et Morales, au sextus;

Congregati sunt (7 v., 1579); ost. si Deus pro nobis, quis contra nos au ténor;

Dignare me (7 v., 1579); ost. au quintus.

En ce qui concerne les messes, on donne une liste plus succinte. En effet, le dépouillement de toute la production du XVIe s. n'a pas encore été fait dans un sens analytique, et la recherche de tous les passages présentant un ostinato dépasse de beaucoup le cadre de cet article. On se limite donc à quelques titres connus.

Pierre Moulu: $\quad$ Missa Stephane gloriose, Agnus III (inédit); ost. en canon aux deux tenors;

Ludwig Senfl: $\quad$ Missa Per signum crucis, Sanctus; ost. au ténor; Das Erbe deutscher Musik, Abteilung Motetten und Messen, Band 1, p. 105;

Johannes Galliculus: Missa Christ ist erstanden, séquence de Pâques; ost. au bassus; Das Chorwerk $\mathrm{n}^{\circ}$ 44, p. 19.

Ciprien de Rore: $\quad$ Missa Vivat felix Hercules (cf. p. 55);

Cr. de Morales: $\quad$ Missa de Beata Virgine, Benedictus; ost. au cantus; Opera omnia, vol. 1, p. 26;

Missa Quaeramus, Agnus II; ost. au ténor I; ibid., p. 188;

Missa l'Homme armé (5 voix), Sanctus; ost. (incipit de la chanson) à l'altus II; ibid., p. 224;

Missa Ut re mi fa sol la, Hosanna; ost. (hexacorde asc.) à l'altus; vol. 7, p. 53; 
Missa Desilde el caballero, Benedictus; ost. en canon aux deux tenors; ibid., p. 79;

Missa Si bona suscepimus, Hosanna; ost. (ré fa mi do ré mi fa) au cantus II; ibid., p. 306;

Missa Ave Maria, Kyrie II; ost. ave Maria au ténor II; vol. 3, p. 34; Missa Tu es vas, différents extraits; ost. tu es vas...; vol. 6, p. l et suiv. ; Missa l'Homme armé (4 voix), Benedictus; ost. (fragment de la chanson) à l'altus; ibid., p. 83; Missa Tristezas me matan, différents extraits; ibid;

Bart. de Escobedo: Missa Philippus rex Hispaniae (cf. p. 55);

G. P. da Palestrina: Missa l'Homme armé (5 voix), Benedictus; ost. au cantus (incipit de la chanson); Opera omnia, ed. F. X. Haberl, vol. 12, p. 99.

Claude Le Jeune: $\quad$ Missa ad placitum, Sanctus; ost. de 4 notes au cantus II; ed. Heugel, p. 33;

T. L. de Victoria: Missa de Beata Virgine, Benedictus, calqué sur celui de Morales; ost. au superius; Opera omnia, vol. 1, p. 89;

Missa Gaudeamus, (probablement inspirée de Josquin) différents extraits; ibid.

L'examen de cette courte énumération révèle la filiation de nombreuses pièces avec les modèles josquiniens (Salve, Gaudeamus, Miserere) et la persistance de quelques motifs à travers les œuvres, comme l'Homme armé ou Ave Maria. Il s'agit là de mélodies ou d'incipits très célèbres, que tout compositeur se plaît à traiter, comme en un exercice de style ou plus précisément, dans le contexte rhétorique où baigne la culture de l'époque, pour honorer un "lieu commun" de l'invention créatrice. Dans ce cas, le but est peut-être de jouer avec la reconnaissance, la recherche (ricerca) du motif par l'auditeur, et l'histoire montre à ce propos une certaine évolution des mentalités au cours du XVIe s., comme on le voit au chapitre suivant; de la recherche de ce qui est caché, on passe volontiers à la manipulation affective de l'auditeur par le jeu de répétitions distinctes. Enfin, la liste précédente met au jour l'emprunt parfois complet d'une œuvre déjà ancienne par un compositeur plus récent (par exemple le Benedictus de la Missa de Beata Virgine, copié par Victoria sur celui de Morales). Pour choquant qu'il paraisse, ce type d'emprunt est chose courante à l'époque et témoigne plutôt d'une certaine admiration pour l'œuvre d'origine; il s'agit là d'un travail d'après modèle, proche de la parodie. Le "contrafactum" rend hommage à la pièce-mère et la magnifie.

\section{Classifications et analyses des pièces baties sur ostinato}

L'aspect monolithique des tableaux précédents cache une grande diversité de réalisation technique. Tout comme le motet isorythmique, la pièce construite sur une partie vocale obstinée 
peut présenter des aspects très variés. Différents paramètres sont alors mis en cause, comme la voix choisie pour l'ostinato, la durée de celui-ci, sa fréquence, sa stabilité ou au contraire ses modifications de hauteurs ou de rythme, sa notation d'origine, le texte traité, et enfin le mode d'élaboration du motif et son sens. En l'absence de sources anciennes suffisamment explicites ${ }^{28}$, certains musicologues cités plus haut ont effectué quelques classifications originales. Il est bon de les rappeler ici, tout en essayant d'en faire une synthèse et de les étoffer. L'établissement d'un "patron" analytique permettra, ensuite, d'étudier en détail les onze pièces de Francisco Guerrero (au chapitre III).

1. Classifications récentes. On dispose essentiellement de deux classements, réalisés par Georg Reichert ${ }^{29}$, et par Willem Elders ${ }^{30}$. Hans Heinrich Eggebrecht ${ }^{31}$, puis Ernst Apfel ${ }^{32}$ ont repris ces modèles sans les agrandir notablement. Enfin, le travail récent de Frédéric Billiet sur le cantus firmus ${ }^{33}$ précise certaines caractéristiques du motif traité en ostinato.

Dans tous les cas, les distinctions suivent (seulement) les aspects généraux de ce dernier. Georg Reichert différencie trois types d'ostinato, en mêlant particularités mélodiques du motif et insertion de celui-ci dans le travail contrapuntique:

1) motif imitant les trompettes ou les cloches; il s'agit de l'imitatio soni tubarum vel campanarum, évoquée dès 1477 par Johannes Tinctoris dans son traité de contrepoint ${ }^{34}$; il distribue les redictae ou répétitions dans chaque voix, sans individualiser d'ostinato. Dans le répertoire, on rencontre néanmoins nombre de pièces illustrant bien le choix de Reichert -par exemple les œuvres de Dufay citées plus haut;

2) motif traité comme un bref cantus firmus, préexistant ou non, souvent répété par transposition au ton et lié symboliquement au texte littéraire. Cette seconde catégorie englobe la grande majorité des cas. Mais les caractères requis paraissent un peu trop abondants et difficilement séparables de l'espèce précédente. Comment nier, en effet, qu'un motif trumpetum (issu de la première rubrique) ne puisse être lié par quelque symbole au texte traité, lui aussi? C'est pourtant le cas dans les Glorias où l'imitation des cloches rappelle les cymbala iubilationis évoquées par W. Elders ${ }^{35}$;

3) pertinacia ou «contrepoint répétitif autour d'un simple cantus firmus», d'après la définition donnée par Zarlino ${ }^{36}$. On ne comprend pas bien l'utilité d'une telle catégorie, car la

28. Les seules pistes analytiques -ouvertes en particulier par Florentius de Faxolis et Zarlino à la Renaissance- ne conduisent à aucune classification générale ni n'offrent de vue d'ensemble sur la technique de l'ostinato. On les explicite néanmoins dans ce chapitre, au fur et à mesure.

29. Ostinato, in: MGG 10, 1962, col. 452-459.

30. Op. cit. 1968.

31. Ostinato, in: Riemann Musiklexikon (1967), pp. 693- 695.

32. Op. cit. 1976.

33. Op. cit. 1999

34. Liber de arte contrapuncti, 1477, ed. Albert Seay, AIM, 1975, p. 152.

35. Op. cit. 1969 , pp. $92-95$.

36. Istituzioni harmoniche 1558 et 1573 (pp. 235-237). On donne la traduction de Reichert entre guillemets, car elle paraît un peu restrictive (lire la suite du paragraphe ci-dessous). Le texte de Zarlino est fort célèbre; mais il n'est pas inutile d'en citer un extrait significatif (op. cit. 1573, p. 236): 
définition zarlinienne, très générale, englobe plusieurs formes d'ostinato. Le texte théorique a peut-être été lu un peu rapidement ou bien sans tenir compte des exemples donnés par l'auteur. La pertinacia (ou, grosso modo, l'ostinato) concerne toute espèce de motif, cantus firmus ou chant figuré, et peut se placer dans n'importe quelle voix. On ne parle donc pas seulement de contrapunto con obligo autour d'un cantus firmus ${ }^{37}$, mais aussi du contraire ${ }^{38}$. Le distinguo opéré par Reichert entre ses rubriques 2 et 3 est donc un peu abusif et son adaptation de la définition zarlinienne assez restrictive ${ }^{39}$.

Ce premier classement manque un peu de clarté. En revanche, celui de Willem Elders est plus précis. Comme on va le voir, son travail reste très partiel, car il s'attache beaucoup à la présentation du motif et à son sens, symbolique ou autre, mais pas à la technique contrapuntique elle-même. En revanche, par souci de rigueur, il élimine d'emblée l'ostinato fugitif et l'ostinato bâti autour d'une voix principale (cantus firmus ou autre). Il est donc l'un des premiers à caractériser clairement un mode de composition où la voix obligée est répétitive; il s'agit d'un cas particulier ou d'une des dernières manifestations de l'isorythmie à la Renaissance, sous forme de motet ou d'extrait de messe sur ostinato. Elders distingue ${ }^{40}$ :

1) l'ostinato simple, an sich. Il place sous cette accolade la plupart des pièces où l'on répète un bref cantus firmus, sans autre précision. Comme exemple prestigieux, il cite le Miserere à 5 voix de Josquin et montre, à travers deux sources critiques de l'époque, l'importance de ce type de composition -considéré comme très difficile ${ }^{41}$ - au début du XVIe s. Les exemples de Josquin et ses contemporains marquent beaucoup les musiciens, et le Miserere est, selon Glaréan 42 "entre les mains de tous" (in omnium manu). Elders en profite pour aborder la portée psychologique et symbolique de l'ostinato; il en sera question plus loin. Tous ces témoignages

"... li Musici si sogliono obligare di fare il contrapunto; usando sempre un passagio, variando pero il concento: il qual modo è detto Far contrapunto con obligo; \& tali repliche o passagi si chiamano Pertinacie: pero quando alcuno si vorrà obligare ad una cosa simile, piglierà à un Thema, o passagio, \& incominciera. a fare il contrapunto sopra il proposto Soggetto... usando hora le Brevi, hora le Semibrevi, hora le Minime, \& le altre figure, lequali potrà porre hora sincopate, \& hora senza la sincopa" (Les musiciens ont l'habitude de s'obliger à faire le contrepoint en utilisant sans cesse un passage, tout en variant les voix environnantes. Ce procédé s'appelle "faire le contrepoint avec obligation", et de telles répétitions ou passages s'appellent pertinacie (= ostinatos). Si quelqu'un désire se contraindre à réaliser une telle chose, il choisit / d'abord / un thème ou un passage et commence à faire le contrepoint sur le sujet proposé... en se servant tantôt des brèves, tantôt des semibrèves, tantôt des minimes et d'autres figures, qu'il peut placer tantôt en syncope, tantôt sans syncope).

37. Voir le premier exemple de Zarlino, op. cit. 1573, p. 237.

38. Voir le second exemple de Zarlino (ibid.). Cette fois, c'est le cantus firmus qui est traité sous forme d'ostinato (varié rythmiquement et transposé); l'autre partie est libre.

39. Hans Heinrich Eggebrecht est plus clair dans sa classification quadruple (op. cit. 1967). Il prend soin de différencier le motif traité sous forme de cantus firmus de ce qu'il appelle ostinate Gegenstimmen ou parties contrapuntiques évoluant passagèrement en ostinato (comme le bassus de l'Et resurrexit de la Missa Ave regina caelorum d'Obrecht).

40. Op. cit. 1968 , pp. 75 et suiv.

41. Zarlino, op. cit. 1573 p. 236 : "questa maniera di far contrapunto è molto difficile" (cette facon de réaliser le contrepoint est très difficile).

42. Heinrich Glarean, Dodekachordon (1547), p. 320. Présentant le mode hypoéolien, il dit: "Nunc eius exempla tractanda sunt. Sed ante omnia ea compositio, quam Iodocus Pratensis super Psalmum Miserere mei Deus: quinque instituit vocibus, palmam obtinet... haec cantio in omnium manu est" (A présent, il faut aborder les exemples de ce /mode/; et cette composition que Josquin des Prés a élaborée à 5 voix sur le psaume Miserere mei Deus obtient la palme avant tous les autres / exemples / . . . cette pièce vocale est entre les mains de tous). 
sont très précieux; on regrette seulement que l'auteur n'établisse pas de sous-groupes pour mieux expliciter les différentes techniques de réalisation;

2) ostinato issu des syllabes de solmisation. En explorant plus intimement la conception de la voce ostinata, Elders met en évidence un type de mélodie relativement courant à l'époque, le sujet élaboré à partir de la sonorité des syllabes solmistiques. En d'autres termes, les voyelles du texte littéraire sont mises en correspondance avec celles des six syllabes de l'hexacorde, ut re mi fa sol la. C'est ce que Zarlino appelle soggetto cavato dalle vocali ${ }^{43}$. Cette technique est très ancienne; un tel rapprochement, un tel jeu sonore laisse des traces dans la musique occidentale depuis fort longtemps -dans l'hymne à Saint-Jean, par exemple. Sur le plan théorique, l'utilisation des syllabes solmistiques dans l'invention contrapuntique semble déjà présente dans les écrits à la fin du XVe s., comme en témoigne le traité de Florentius de Faxolis ${ }^{44}$. Dans la pratique, l'œuvre de Josquin fournit là encore des exemples prestigieux. Elders cite bien sûr le sujet de la Missa Hercules dux Ferrariae, re ut re ut re fa mi re, et aussi le motif la mi la, répété par le ténor dans le motet Illibata Dei virgo nutrix. Cette fois, la solmisation se suffit à elle-même et les associations avec des mots adéquats comme Maria sont invérifiables, mais tout à fait séduisants. Il semble qu'on entre alors dans le jeu subtil des rébus et énigmes proposés à la sagacité des chantres, dans un contexte quelque peu corporatiste. C'est le domaine dévolu à la rubrique suivante;

3) ostinato explicité par une "devise" ou canon. Elders s'attache ici à la dimension symbolique du lien unissant le motif, son texte et celui du canon. Ce dernier peut, par exemple, induire un jeu rythmique (crescite et multiplicamini) ou bien montrer une route mélodique à suivre (vade retro Satanas). Le jeu d'énigmes est alors de règle. Dans le contexte où travaille Elders -l'étude du symbolisme dans les œuvres des Franco-flamands- cette rubrique prend tout son sens. En revanche, elle n'apporte rien de bien nouveau sur le plan technique.

Comme on le disait au début de ce chapitre, les deux classifications précédentes ne sont pas complètes et laissent de côté un certain nombre d'aspects pratiques. Hans Heinrich Eggebrecht, qui écrit presqu'en même temps que Willem Elders, entrevoit quatre catégories différentes ${ }^{45}$ mais

43. Op. cit. 1573 , p. 329: "bisogna sapere che tal Soggetto puo essere fatto dal Compositore; come fece Giosquino il Tenore di La sol fa re mi; \& il Tenore della Messa Hercules Dux Ferrariae, cavato dalle vocali di queste parole" (il faut savoir qu'un tel sujet peut être façonné par le compositeur lui-même, comme l'a fait Josquin pour le ténor de / la messe / La sol fa re mi et pour le ténor de la messe Hercules dux Ferrariae, élaboré à partir des voyelles de ces paroles-là).

44. Liber musices (ms., circa 1496; Milano, Biblioteca Trivulziana, codex 2146). Au chapitre 16 (p. 88), on lit, à propos du canon: "Aliquando verborum canonis vocales litteras vel syllabas vel dictiones notabimus, in quibus diversas figuras collocando, cui ipsorum competant eae, experiemur" (Parfois, nous relèverons des voyelles ou des syllabes ou bien des morceaux de mots du canon, sur lesquels nous nous exercerons en y plaçant diverses figures / musicales / qui puissent correspondre au / canon / de ces /mots / eux-mêmes). Si l'on prend soin de saisir le terme "canon" dans son sens originel, c'est-à-dire la règle ou devise permettant d'interpréter le contrepoint en imitation continue, cette phrase peut révéler une prise en compte fort ancienne des jeux solmistiques dans la composition. Les syllabes auxquelles Florentius applique des figures de notes peuvent-elles représenter autre chose que des syllabes de solmisation? Cela semble peu plausible.

45. Op. cit. 1967. Le musicologue distingue (p. 694): l'ostinato descriptif (cloches...), l'ostinato sous forme de bref cantus firmus, l'ostinato solmistique (cavato dalle vocali), et enfin les ostinate Gegenstimmen ou voix concomitantes obstinées. On ignore l'influence que cette liste a pu avoir sur W. Elders, qui publie un an après. Toujours est-il que, depuis l'article de G. Reichert analysé ici (1962), celle-ci s'est agrandie et affinée. L'année 1967 marque un regain d'intérêt pour une réflexion de cet ordre. 
n'est pas plus précis, contrapuntiquement parlant. Seul, l'Abbé R. B. Lenaerts apporte un peu de lumière sur cette question ${ }^{46}$, en différenciant deux modes de répétition du motif:

1) répétitions identiques, avec seulement la possibilité de transposer l'ostinato sur d'autres hexacordes;

2) répétitions avec transposition degré par degré, suivant le modèle du Miserere à 5 voix de Josquin.

On tient compte de ce judicieux distinguo dans les lignes qui suivent. Mais, pour tenter d'être complet, il reste à évoquer l'aspect rythmique, jusqu'ici contourné, et à s'interroger sur le sens de ce mode de composition. Là, les travaux d'Elders sont, il est vrai, d'un grand intérêt.

2. Etablissement d'un "patron” analytique. Il est nécessaire, avant d'aborder les œuvres de Guerrero, de faire la synthèse des données précédentes et de les étoffer. On ne cherche pas à établir une nouvelle classification, mais plutôt à répertorier les différents paramètres d'analyse conduisant à une bonne appréciation des partitions. Dans ce but, on énumère les éléments techniques les uns après les autres, en rappelant si nécessaire leur présence dans les rubriques musicologiques explicitées plus haut. Pour chaque ostinato, il paraît bon d'observer:

-le texte traité (et le texte des autres voix, s'il y a lieu);

-la partie vocale choisie;

-la mélodie, son origine et son mode d'élaboration;

-la notation originale de la voix, qui forme l'architecture de la pièce par l'intermédiaire du nombre de répétitions, de leurs dimensions (en termes de temps musical) et de leurs techniques de réalisation (transpositions, mises en proportions, modifications mélodiques ou contrapuntiques); une typologie très riche apparaît alors, comme on le voit plus loin;

-l'insertion de l'ostinato dans la polyphonie, qu'il entre dans le jeu des entrées ou qu'il reste indépendant;

-les compléments d'analyse "sémantique", permettant de préciser la portée de la pièce en faisant appel à différents aspects de la culture ancienne comme la rhétorique.

C'est d'ailleurs là que cette étude prend toute sa signification. Il s'agit d'étendre le travail du seul domaine largement exploré par Elders - la symbolique- aux régions plus vastes de l'architecture, des mathématiques et de l'art oratoire. La musique se liant à cette époque autant aux arts du trivium qu'à ceux du quadrivium, il est vraiment nécessaire de rassembler toutes les données afférentes en un vaste tableau d'essence humaniste.

Pour cela, on détaille chaque paramètre donné ci-dessus. Par souci de clarté, les éléments d'interprétation culturels dont on vient de parler complètent l'analyse au fur et à mesure.

-Le texte. On distingue deux cas: A) le texte de l'ostinato correspond à l'incipit de celui des autres voix; la pièce est monotextuelle; B) il est différent et la pièce est bitextuelle.

46. Zur Ostinato-Technik in der Kirchenmusik der alten Niederländer, in: Festschrift Bruno Stäblein, Kassel, 1967, pp. 157-159. 
Le texte peut provenir de deux "langages" différents: a) il est littéraire, ce qui est très courant; b) il est formé de syllabes solmistiques, ce qui est rare (exemple: la mi la dans le motet Illibata de Josquin).

Le texte, s'il est littéraire, peut être profane ou sacré, rédigé en latin ou dans une langue vernaculaire. Il peut avoir différents caractères ou remplir différents rôles; les musicologues allemands ${ }^{47}$ évoquent ainsi 1'appel, le cri (Ruf) -par exemple Lazare veni foras chez Clemens non Papa et Lassus ${ }^{48}$, la prière (miserere mei Deus), la devise (die Devise), par exemple nosce teipsum (connais-toi toi-même) chez Lassus ${ }^{49}$, et l'apostrophe ou salut (ave Maria), etc. A ce propos, un premier rapprochement entre composition musico-poétique et art héraldique paraît d'un grand intérêt. L'art du blason brille de tous ses feux à la fin du moyen-âge et occupe une bonne place dans le monde culturel humaniste. Il sert même de modèle à certains types de poèmes comme le "blason". Sa langue très typée et ses règles peuvent être d'un grand secours pour mieux appréhender certains phénomènes artistiques. En musique, on en parle assez peu, en général. Et pourtant, comment ne pas mettre en parallèle, dans le cas présent, la devise ou le cri de l'ostinato avec les mêmes termes, issus de l'art héraldique? Comme le rappelle Léon Jéquier ${ }^{50}$, "la devise (placée au-dessous du blason) était accompagnée du "mot", âme de la devise. Ce "mot" est formé d'une ou plusieurs lettres, d'un mot ou de toute une phrase... Les mots, actuellement appelés devises, ont le plus souvent trait à l'histoire, à la patrie ou à la religion... Le cri servait de signe de ralliement et de bataille".

La brièveté, le caractère oratoire invitatif, implorant et de toute façon très engagé de ces cris ou devises est très proche de l'appel laconique et réitéré de l'ostinato musical. L'aspect itératif de ce dernier a quelque chose à voir avec le cri lancé à mainte reprise sur le champ de bataille. On décèle ainsi quelqu'analogie de fonction entre ces deux types de devises, et l'emploi du terme par les musicologues germaniques n'est certainement pas fortuit. Un tel rapprochement situe mieux le rôle de la pièce en ostinato: il s'agit de rappeler sans cesse la direction, le sens donné à l'acte religieux, en s'adressant au saint auquel l'office est dédié, ou bien en plaçant en toile de fond l'écho d'un appel lié au climat dans lequel s'inscrit le texte des autres voix. C'est ce qu'Elders nomme Hintergrund (arrière-plan). L'ostinato agit alors comme une horloge sémantique sonnante, amplifiant 1'idée par répétition jusqu'à l'exaltation.

Ceci permet d'inscrire cette technique dans le contexte rhétorique sans difficulté. En effet, la répétition est l'un des moteurs essentiels de l'ornementation oratoire. Et, lorsque les théoriciens commencent à s'intéresser de près aux rapports entre rhétorique et musique (dès le milieu du XVIs s.), ils n'hésitent pas à classer diverses formes de répétitions musicales dans leurs listes de figures calquées sur celles de l'art oratoire antique. Au-delà des climaces et palillogiae de Burmeister ${ }^{51}$, un auteur comme Johannes Nucius place précisément sous le signe de la figure

47. Voir en particulier W. Elders, op. cit. 1968, pp. 75-85.

48. Motets Fremuit spiritu Jesus, cités plus haut.

49. Motet Homo cum in honore, cité plus haut.

50. Manuel du Blason, d'après D. L. Galbreath, Lausanne, Spes, 1977, pp. 211-214.

51. Joachim Burmeister, Musica poëtica, Rostock, 1606, pp. 63-64. 
repetitio la technique de 1 'ostinato héritée du XVe $\mathrm{s}^{52}$. C'est dire l'importance que ce type de composition possède dans le discours musical épidictique et la portée affective qui est alors la sienne $^{53}$. Puisque la répétition permet de "mieux faire entrer la sentence dans les esprits" 54 , le sens rhétorique de l'itération paraît bien être pris en compte par le compositeur: c'est un mode d'expression codifié, visant à amplifier un (bref) message, répété d'une facon incantatoire. Et, pour en revenir à l'héraldique, on jette à cette occasion un pont entre cet art et la rhétorique. La figure exclamatio ou ekphonesis est présentée par certains théoriciens ${ }^{55}$ comme un cri ou une devise. L'appel de 1'ostinato paraît ainsi doublement figuraliste, de par son caractère répétitif (figure repetitio), et de par son caractère héraldique.

Le texte et la réalisation de l'ostinato peuvent être introduits par une règle ou "canon", rédigée en latin ou dans une langue vernaculaire. Cette courte introduction précise alors le sens donné à la pièce ou bien la technique contrapuntique mise en jeu. Ainsi, le motif obstiné du motet Domine Deus noster de Josquin porte la devise Crescite \& multiplicamini (Croissez et multipliez). Le "canon" donne la clé de l'interprétation rythmique du sujet, qui doit être traité en proportion à chaque répétition. La devise permet, comme le dit Elders, d'établir un lien entre 1 'idée de l'ostinato et la composition ou bien entre l'idée de l'ostinato et la devise elle-même ${ }^{56}$. Ainsi, la Missa Adieu mes amours de Andreas de Silva porte le canon suivant, au Sanctus: Dinumerabo nomen tuum in eternum (Je compterai ton nom pour l'éternité). Elders pense avec raison que cette expression induit l'idée de la répétition continuelle ${ }^{57}$. De même, le motet Tu es Petrus de Simon Moreau porte, dans la version d'Hermann Finck, la règle suivante pour le discantus: Itque reditque frequens, non qui inceperit, sed qui perseveraverit (Il va et revient en se répétant, pas celui qui a commencé, mais celui qui s'est obstiné). C'est peut-être la meilleure présentation possible de l'ostinato ${ }^{58}$. Ici, le rapport entre texte introductif et musique est sémantique et parfois symbolique. La rhétorique n'intervient que dans la forme de la devise ellemême, qui peut comporter des jeux de mots (paronomases) de rhétoriqueurs ou des tropes de

52. Johannes Nucius, Musices poeticae, Neisse, 1613, $\mathrm{f}^{\circ} \mathrm{G} 2$ : "Quid est Repetitio? Cum in Contrapuncto florido seu fracto, thema in una aliqua voce perpetuo iteratur, quamtumvis mutatis locis. Exempla huius Schematis sunt in Missa super La Sol fa re mi. In Miserere mei Deus. In fremuit Spiritus" (Qu'est-ce que la répétition? / Il y a repetitio / en contrepoint fleuri ou brisé, lorsqu'un thème est continuellement répété dans une même voix, et si l'on veut en des lieux différents. On trouve des exemples de cette figure dans la messe sur La sol fa re mi, dans le Miserere mei Deus /de Josquin/, dans Fremuit spiritus /de Clemens et Lassus/.

53. W. Elders rapporte ainsi les paroles de l'éditeur Hans Ott, dans la Préface du Novum \& insigne opus musicum (Tome II, Nurnberg, 1537). A propos du Miserere de Josquin, cet auteur dit que l'ostinato est "une répétition très grave par laquelle il implore la miséricorde"(gravissima repetitio qua misericordiam implorat).

54. J. Burmeister, op. cit. 1606, p. 73.

55. Par exemple J. C. Gottsched (Ausführliche Redekunst, Leipzig, 1736, p. 286), ou J. Mattheson (Der vollkommene Capellmeister, Hamburg, 1739, p. 193); cités par Dietrich Bartel, in: Handbuch der musikalischen Figurenlehre, Laaber, Laaber-Verlag, 1997, pp. 150-151.

56. Op. cit. 1968 , p. 84.

57. La notation de la partie vocale permet, seule, de savoir si cette devise n'apporte qu'une précision à une exécution musicale explicite ou bien si elle en constitue la clé. Voir plus loin ce qui concerne l'élaboration de l'ostinato.

58. H. Finck, Practica Musica, Witenberg, 1556, f ${ }^{\circ}$ Cc. L'auteur explicite ainsi la devise: "Significat, cantilenam, absoluta serie notarum, iterum abque iterum ab initio repentendam, donec reliquae voces etiam cessent" (Cela signifie que la cantilène / du superius / doit être répétée à plusieurs reprises depuis le début, suivant la série de notes toute entière, jusqu'à ce que les autres voix s'arrêtent). C'est une description très claire du phénomène, qui servira de guide dans le prochain alinéa. 
toutes sortes. Plus marquant est l'apport de l'héraldique, qui fait surgir, là encore, un parallèle entre rédaction du canon et ornementation du blason. Le laconisme de la devise, son expression stylisée proche de l'aphorisme ou du proverbe, son rapport à l'histoire ou à la religion la situe sur le même plan formel que le "mot" héraldique, comme on l'a vu précédemment, en citant L. Jéquier. Son désir de cacher la réalité, son caractère sybillin sont aussi ceux du cri du héraut, qui, selon $\mathrm{O}$. Neubecker ${ }^{59}$, cultive à la Renaissance une expression quelque peu dissimulée. Enfin, la terminologie autorise des rapprochements troublants. Le "canon" gréco-latin des théoriciens impose une définition très technicienne de la tournure compositionnelle implicite -une règle contrapuntique-, tandis que bien des traités emploient plutôt le terme latin titulum $^{60}$ ou le mot allemand Spruch ${ }^{61}$, qui se traduisent par "devise" et servent également à désigner le cri ou la maxime héraldique. En Italie ${ }^{62}$, Ramos de Pareja parle de subscriptio (indication souscrite) et Gaffurio de sententia (sentence, maxime). Sans être aussi transparents, les liens qui unissent ces termes au blasonnement sont fort instructifs. Un véritable art de l'expression ramassée, sémantiquement riche, volontiers élitiste, paraît ainsi réunir des mondes bien différents comme la pièce musicale et le blason, mais certainement très proches dans le quotidien et le mode de pensée des hommes, en ce temps-là.

-Le choix de la voix traitée en ostinato constitue un paramètre analytique non négligeable. On écrit voix au singulier, en rappelant que les duos sont rares en ce domaine (l'Agnus III de la Missa Stephane gloriose de Pierre Moulu, par exemple). On constate que les compositeurs placent volontiers le motif au ténor ou dans une voix élevée, altus ou, plus souvent encore, superius. Le respect de la tradition, qui donne la primauté au ténor, transparaît dans cette élection; il entre naturellement en lice avec l'usage un peu plus récent qui consiste à s'intéresser davantage au superius ${ }^{63}$. Certaines filiations transparaissent par ce biais, d'un musicien à l'autre. Ici, Guerrero paraît marquer un lien privilégié avec son maître Morales, en plaçant souvent le voce ostinata au cantus $\mathrm{II}^{64}$. Mais, au-delà de ces options techniques traditionnelles, on décèle parfois l'influence du symbole et de la rhétorique dans certaines dispositions vocales. Ainsi, les motets mariaux mettent volontiers l'ostinato dans une voix aigue, en une sorte de "paronomase" musico-architecturale ${ }^{65}$. En analysant les pièces de Guerrero, on constatera aussi que le motif s'entend plus nettement que dans nombre d'autres œuvres. Le souci oratoire de clarté, de persuasion, d'efficacité semble guider le choix du compositeur. La figure repetitio, écart sensible

59. Le grand Livre de l'Héraldique, Bruxelles, Elsevier Séquoia, 1977, p. 212: "A l'époque de l'Humanisme, les lettrés ont rivalisé dans l'invention de rébus impressionnants / pour la devise /, dont le déchiffrement était rarement possible du premier coup... / En Italie /, l'image est accompagnée d'une inscription sur une banderole, souvent à caractère ésotérique". 60. Terme employé par exemple par Seth Calvisius en 1592 (Melopoiia, Erfurt, 1592, f I 5) ou par J. A. Herbst (Musica poëtica, Nurnberg, 1643, p. 95).

61. Voir J. A. Herbst, op. cit. 1643, ibid. En allemand, le terme Spruch désigne une courte maxime (profane ou sacrée) ou bien un verset que l'on inscrit dans le phylactère de la devise placée sous le blason.

62. Ramos de Pareja, Musica practica, Bologna, 1482, tertia pars, tractatus primus, cap. 4, p. 90 dans la nouvelle édition allemande (Breitkopf, 1968). F. Gaffurio, Practica Musice, Milano, 1496, f ${ }^{\circ}$ cc iiij.

63. Voir par exemple la démonstration de Nicolo Burzio, in Musices opusculum, Bologna 1487, fo e 6. A 1 'époque baroque, les musiciens placeront l'ostinato au bassus.

64. Voir chapitre III.

65. C'est le cas, par exemple, dans le Salve regina à 5 de Josquin. 
dans le langage polyphonique, atteint son maximum d'intensité en étant placée dans la voix supérieure qui, selon Zarlino, "est plus pénétrante pour l'ouie"66. La balance entre volonté de cacher le procédé et, au contraire, d'en extravertir l'effet incantatoire au maximum marque bien le passage à 1'esthétique baroque au cours du XVIe s. Encore que ces propos puissent être nuancés par les usages d'interprétation. W. Elders penche pour l'introduction de cloches dans de telles pièces, pour assumer la partie répétitive ${ }^{67}$. Dans ce cas, le jeu rhétorique de la disposition vocale s'effondre. Mais une telle pratique instrumentale n'est ni prouvée ni conçue universellement.

-Elaboration du motif ${ }^{68}$. Le sujet a généralement la forme d'un incipit de cantus firmus, traité en valeurs longues (de la longue à la semibrève ou à la minime). Il comporte parfois une portion terminale ornée ${ }^{69}$. Le style prosodique n'est pas toujours syllabique: les mélismes s'étendent sur les notes allongées comme sur les valeurs rapides. Le motif peut être d'origine solmistique (soggetto cavato dalle vocali), complètement ou partiellement ${ }^{70}$. Ce peut être un timbre connu, généralement sacré (Ave Maria, Gaudeamus, Pater noster, etc.), qui porte ou non son texte d'origine; dans ce dernier cas, la mélodie s'adapte au texte (de la messe, le plus souvent) qui lui est imposé. Le rappel du motto célèbre agit, suivant W. Elders, par son poids psychologique et sa force évocatrice des paroles initiales ${ }^{71}$. Un lien subtil s'établit comme cela entre la musique de l'office et le contexte de celui-ci. Ainsi Guerrero amplifie la prière adressée à la Vierge et la glorifie à chaque reprise de l'incipit Sancta et immaculata, dans le Sanctus de la messe du même nom ${ }^{72}$.

-La notation de l'ostinato et l'architecture de la pièce sont indéfectiblement liées. Si l'on veut en percer les secrets de fabrication, l'étude de la notation d'origine est indispensable. Le jeu fondamental reposant sur l'alternance entre silence et répétition vocale, il est nécessaire de savoir comment le compositeur conçoit cette succession. Son écriture doit faire apparaître la ou les périodicités correspondantes. On découvre que le temps musical y est règlé d'une façon très précise. La disposition fait montre d'un strict proportionnement des évènements sonores. Leur durée répond à cette "tendance à construire le cantus firmus en proportions mathématiques" "73, forte chez les compositeurs du temps, pour qui "les proportions numériques jouent un rôle très grand, dominant"73. Les différents jeux de hauteurs et de divisions arithmétiques

66. Op; cit. 1573 , p. 281.

67. Op. cit. 1969.

68. Les musicologues germaniques disent également Motto, Thema (W. Elders), Devise (L. Finscher, W. Elders).

69. Voir, par exemple, T. L. de Victoria, Missa de Beata Virgine (1576), Benedictus; le motif du superius s'achève par une cadence ornée.

70. On peut en effet considérer que le lien intellectuel entre notes de musique et voyellessyllabes solmistiques déborde largement le cadre précis du soggetto cavato. Ainsi le sujet du Miserere de Josquin est en accord avec les syllabes sur les notes extrêmes de la principale présentation du sujet (la syllabe "mi" de mi(serere) et le "i" de (me)i, chantés sur E). Il est probable qu'il faille tenir compte plus systématiquement d'un tel rapport structurel entre les deux codes de langage, littéraire et musical. La solmisation pourrait passer pour une sorte de "métalangage", suivant une tenminologie célèbre.

71. Op. cit. 1968, pp. 76 et 82.

72. Voir chapitre III.

73. L. Finscher, op. cit. 1990 , p. 315 . Voir aussi chapitre III, p. 86. 
apparaissent également dans la notation, symboliquement rapportés à des signes divers, de reprise, de mensuration, etc. Cette fois, ce n'est plus tant l'univers de l'art oratoire qui capte notre attention, mais plutôt le lien de la musique avec les arts du quadrivium. Le travail est arithmétique et procède de la géométrie dans l'espace musical.

Le sujet est noté de plusieurs facons, soit explicites, soit elliptiques, avec parfois la volonté de cacher l'ouvrage. En général, les répétitions ne sont pas recopiées intégralement ${ }^{74}$. Le compositeur (ou l'éditeur) donnent seulement le motif, environné des pauses de respiration prélables et/ou consécutives. Dans certaines éditions, comme celle de Guerrero à Paris, en 1566, le nombre d'interventions du cantus firmus est indiqué par un chiffre placé à droite, entre deux barres verticales. En cas de transposition, la mélodie peut être présentée à chaque hauteur correspondante autant de fois qu'il y a de transpositions, ou bien seulement une seule fois, le "canon" ou un signe de prise indiquant la marche à suivre. Il en va parfois de même pour les proportions rythmiques. En fait, il n'y a pas de règle générale; l'éditeur paraît libre de confier tout ou partie de la donnée au lecteur, s'adressant plutôt à des chantres experts. Il est néanmoins possible de proposer ici quelques solutions couramment rencontrées dans les éditions anciennes. Celles-ci tiennent compte de la réunion de quelques paramètres: présence ou absence de silence introductif, transpositions éventuelles, mises en proportion, modifications mélodiques ou rythmiques. Une typologie assez générale découle de leurs diverses combinaisons. On précise, en ce qui concerne les transpositions, que deux cas principaux se rencontrent. En premier vient la transposition hexacordale, la plus fréquente. Le sujet est déplacé d'une seule façon: il monte ou baisse d'une 4te ou d'une 5te, suivant la position de l'hexacorde vrai le plus proche, en passant de "nature" à "bécarre" ou "bémol", par exemple. Parfois, un hexacorde feint est employé; c'est en général ${ }^{75}$ l'hexacorde de B-mol, où l'on lit "ut" sur si bémol. En second lieu vient la transposition par degré, portée aux nues par Josquin dans son Miserere: le motif descend les sept degrés de l'octave modale de $\mathrm{Mi}$, les remonte, puis en redescend quatre. L'idée est remarquable; la double catabase du tenor souligne la chute douloureuse du pécheur. Cet exemple a été suivi mainte fois $^{76}$ et la transposition gradatim ou par pes descendens vel ascendens ("pied" descendant ou ascendant) est l'une des signatures les plus célèbres du travail sur ostinato.

En ce qui concerne les proportions, bien des solutions ont été mises en jeu, de la plus simple à la plus complexe. L'une des plus aisées consiste à diviser les durées par deux, au moyen d'un signe adéquat. Ainsi, la notation d'origine est préservée, et c'est seulement le changement de durée des valeurs qui opère l'accélération au moment de l'exécution ${ }^{77}$. A l'opposé, Josquin pose un grand problème d'analyse à l'interprète avec son canon Crescite et multiplicamini ${ }^{78}$. Il met à rude épreuve la sagacité des chantres. Comme on le verra chez Guerrero, ce type de jeu tend à se raréfier à la fin du XVIe s. ou à se simplifier. L'attention se porte plus, semble-t-il, sur les qualités oratoires de la pièce que sur sa virtuosité mathématicienne.

74. On verra une exception plus loin: le motet Fremuit spiritu de Lassus (Il primo Libro de Motetti a cinque, Antwerpen, 1556).

75. Comme dans le motet Illibata de Josquin.

76. Voir chapitre I.

77. Voir Guerrero, ostinato de la Missa Ecce sacerdos, chapitre III.

78. Motet Domine Deus noster, cité au chapitre I. 
Enfin, les modifications mélodiques sont assez rares, d'un terme de l'ostinato à l'autre. Dans ce cas, elles sont généralement indiquées, tout comme certaines mutations rythmiques. On notera l'impossibilité de quantifier le point d'orgue final qui clôt la voce ostinata. Dans les calculs, il est souvent difficile de prendre sa durée en compte. Comme le dit H. Finck à propos du motet de Simon Moreau ${ }^{79}$, on répète le motif “jusqu'à ce que les autres voix s'arrêtent".

Les combinaisons principales de tous ces paramètres permettent de distinguer au moins quatre modèles différents. Comme on s'attache à la notation d'origine, on différencie en premier lieu les parties vocales notées in extenso (Exemple 1), de celles qui sont écrites d'une façon globale, résumée. Ces dernières peuvent présenter un silence introductif (Exemples 2, 3) ou commencer par le motto (Exemple 4). On oppose enfin un extrait où le motif est énoncé une seule fois (Exemple 2) et un autre où il figure deux fois (Exemple 3), sans que cela corresponde à l'obligation d'une transposition / d'une mise en proportion ou non.

Exemple 1: Présentation de la partie vocale in extenso.

Motet Fremuit spiritu de Roland de Lassus; motif Lazare veni foras (op. cit. 1556).

Noter les modifications rythmiques: valeurs de notes, silences. L'ensemble ne paraît pas structuré d'une façon systématique.

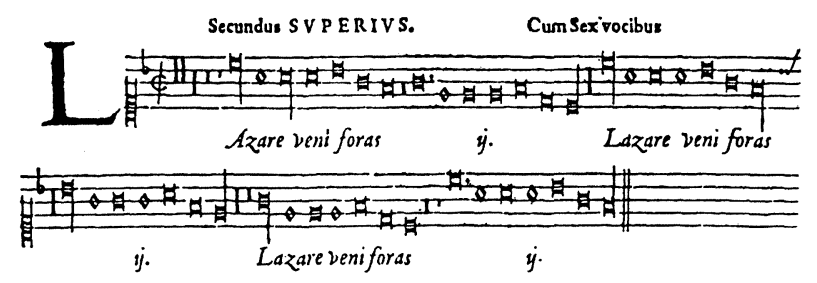

Exemple. 2: Présentation globale d'un seul motif.

Antoine Brumel, messe de Dringhs, Agnus II à 2 voix.

Donné par Glaréan (op. cit. 1547, p. 459).

L'ostinato part dès le premier tactus et le nombre de reprises est indiqué par les quatre syntagmes du texte souscrits. Il n'y a ni transposition, ni proportion.

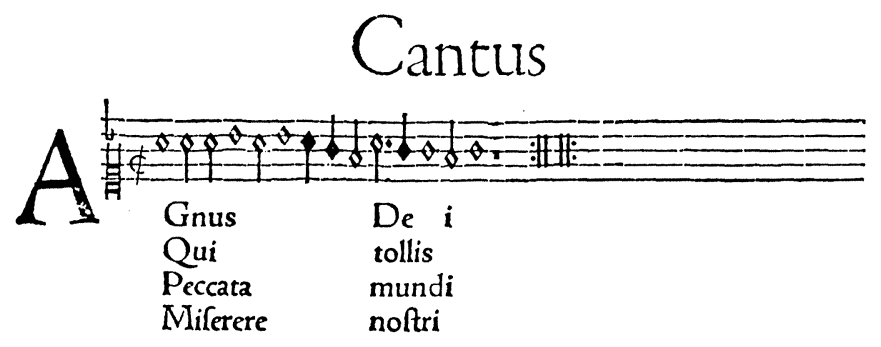

79. Voir plus haut, p. 65. 
Exemple 3: Présentation globale d'un motif sans modification, introduit par un long silence.

Ferdinando de Las Infantas, motet Veni Domine (1579).

On notera la délimitation du silence introductif par une barre verticale. La boucle de l'ostinato s'ouvre donc seulement après ce signe.
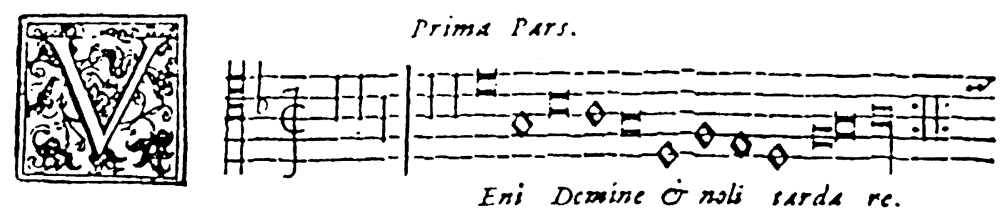

Exemple 4: Présentation globale d'un motif et de sa transposition.

Simon Moreau, motet Tu es Petrus, donné par H. Finck (cf. p. 15).

Ici, les pauses introductives ont la même durée que les pauses intercalaires. On note une certaine "symétrie".

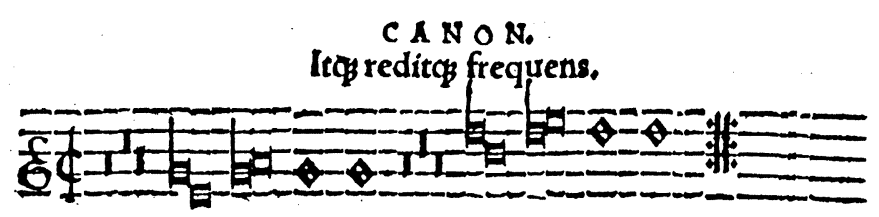

Toute autre est l'apparence des œuvres, une fois mises en abîme. Leur disposition complète s'étale sous les yeux et oblige l'analyste à rechercher des modèles et des concepts qui puissent en rendre compte d'une façon plus générale. Dans ce contexte, le musicologue peut faire appel une dernière fois à l'art héraldique. En effet, la représentation schématique de la voce ostinata manie certains éléments de géométrie. Et, pour les interpréter, il paraît plus cohérent de s'immerger dans le contexte culturel de la Renaissance que de s'attacher aux mathématiques du XXe s. -c'est en tous cas le choix émique opéré le plus souvent dans ce travail. L'art du blason possède une terminologie très riche pour désigner les différentes partitions de l'écu ${ }^{80}$. Les divisions verticales intéressent en particulier le musicien, face au schéma de la partie vocale répétitive, où alternent blocs de notes et de silences. Une analogie s'établit sans peine entre ceuxci, dessinés globalement, et les partitions du blason. En voici un exemple:

80. Voir L. Jéquier, op. cit. 1977, pp. 101-123. 
Exemple 5: Représentation graphique de 1'Exemple 2.

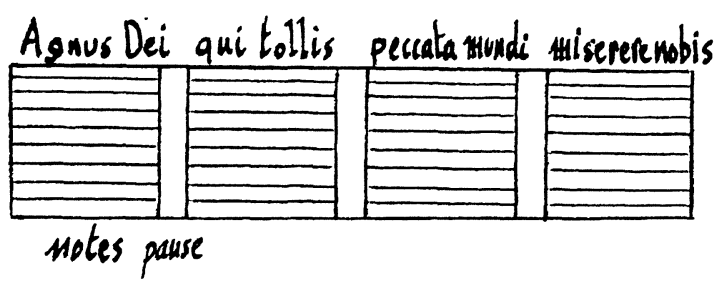

Deux premiers cas de figure se signalent, suivant l'ordre de ces blocs:

- les silences sont intercalaires et notés en blanc (Exemple 6-1);

- il y a un silence initial et des pauses intercalaires. On peut rapprocher immédiatement ces dispositions de leur équivalent héraldique, les "pals" et le "palé" (Exemple 6-2):

Exemple 6: 1) "trois pals" de silence, comme dans l'Exemple 2;

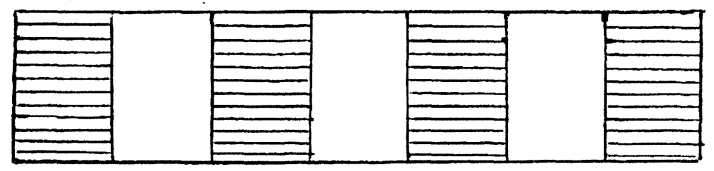

2) "palé de 4 pièces", comme le sujet de l'Exemple 4.

(Notation de la transposition: voir ci-dessous);

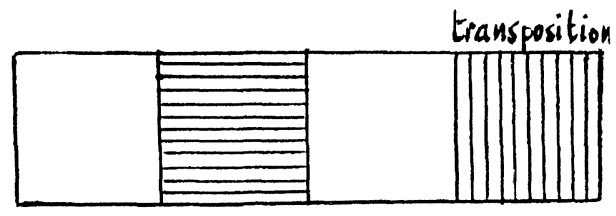

3) si l'on donne cet exemple in extenso, on obtient un palé ou plutôt un "vergeté" de 20 pièces, le sujet chantant 5 fois.

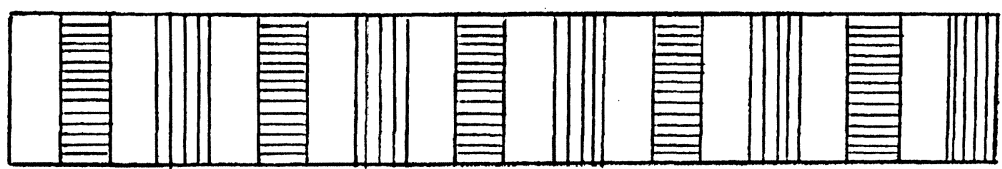

Pour affiner ces schémas, on teinte les pals hachurés de deux "émaux" différents en cas de transposition du motif musical; l'un est rayé horizontalement et l'autre (la transposition) verticalement, suivant les usages héraldiques. Pour représenter le silence introductif, on place un large pal blanc à gauche, qui est dit "adextré". En cas de répartition irrégulière des silences ou 
des notes, les pals sont inégaux. Enfin, s'il y a mise en proportion musicale, on peut séparer le schéma en deux ou plusieurs régions, verticalement. Cela s'appelle le "parti" (Exemple 7). Il est plus ou moins symétrique; s'il est plus grand à gauche, c'est un "senestré", et un "adextré" dans le cas contraire.

Exemple 7: Schéma de l'Exemple 6-2 en "parti senestré", en imaginant que l'oeuvre utilise la proportion double.

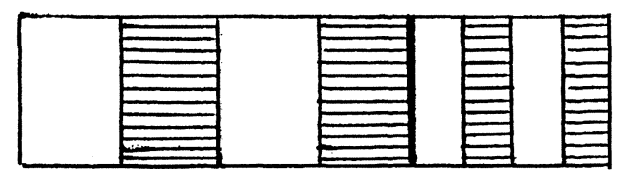

Les graphiques ci-dessus autorisent une analyse arithmétique de la disposition musicale. Des nombres apparaissent, indiquant les proportions des blocs (notes et silences). A ce propos, certaines constantes ont été révélées par les exégètes de notre temps, en particulier dans les pays germaniques. Les travaux de Rolf Dammann ${ }^{81}$, de W. Elders ${ }^{82}$ ou de Gösta Neuwirth ${ }^{83}$ sur la période josquinienne font autorité en la matière. Le cadre de cet article interdisant toute démonstration trop développée, on se contente de rappeler que l'écriture du tenor ou du cantus firmus tend à suivre un mode d'élaboration très sophistiqué à la Renaissance. L'axe organisateur est d'essence arithmétique et met en valeur des nombres, choisis comme étalons de construction des différents paramètres de 1'oeuvre musicale: nombre de voix, de tactus, de silences, de répétitions textuelles, etc. ${ }^{84}$. L'aspect symbolique de ces mensurations peut également éclairer 1'analyse d'une façon certes un peu cachée, mais bien accordée avec le contexte culturel du temps, si tant est qu'on veuille le prendre en compte dans sa globalité. W. Elders évoque ainsi 1'importance du nombre 7 -nombre parfait mais aussi funeste et lié à la remissio peccatorumdans le Miserere de Josquin ${ }^{85}$ et d'autres œuvres du même caractère.

Appliquée aux pièces bâties sur un ostinato, ce type d'analyse donne des résultats non négligeables. La seule difficulté est d'appréhender la dimension de l'étalon-constructeur. En effet, plusieurs possibilités sont offertes: observer d'abord la durée de 1'ostinato lui-même, ou bien y ajouter les silences concomitants, ou bien, en cas de transposition musicale, circonscrire une sorte de "grand module" embrassant la totalité du cycle de notes et de silences. Mais 1'expérience prouve qu'il n'y a pas de solution unique; il est bon d'emprunter diverses pistes avant de retenir une solution, et il est rare qu'on n'aboutisse pas à une donnée numérique normative: nombres $4,6,7,12,19(=12+7)$, multiples de $12(24,36)$, de $19(76=19 \times 4)$, etc. $\mathrm{Au}$ chapitre suivant, on en fait l'essai avec succès sur les onze pièces de Guerrero.

81. Spätformen der isorythmischen Motette in 16. Jahrhundert, AMw X, 1953, pp. 16-40.

82. Op. cit. 1968, en particulier pp. 124-128: Ostinato und Zahl.

p. 21 .

83. Erzählung von Zahlen, in: Musik-Konzepte 26/27, Josquin des Prés, München, 1982, pp. 4-38, en particulier

84. Voir W. Elders, op. cit. 1968, pp. 92-152 (Drittes Kapitel).

85. Le motto y est entendu 21 fois, soit 3 fois 7 , etc. 
Au passage, quelques réflexions sur l'architecture des œuvres s'ouvrent sur les grandes questions artistiques de la Renaissance: y-a-t-il symétrie ou bien recherche d'une symétrie? Notet-on une certaine volonté de mise en perspective (en donnant à l'espace sonore et au temps musical une plus grande dimension par le mélange entre voix à l'élocution rapide et cantus firmus alangui et parlant périodiquement) ? Sans poser ces questions systématiquement, on y fait allusion le cas échéant au chapitre III.

-La construction musico-syntaxique des pièces se limite ici à quelques remarques d'ordre général; le sujet est d'ailleurs fort peu abordé par les musicologues. L'intérêt est de voir comment les compositeurs intègrent l'ostinato dans la trame polyphonique et varient le contrepoint à chaque présentation.

Signalons que le nombre de voix joue un rôle important dans la construction. L'effet du cantus répétitif n'est pas du tout le même à 4 voix qu'à 6 . Plus l'effectif augmente, plus il est facile de cacher l'ostinato et de ménager des contrastes saisissants quand il s'entend à découvert (c'est le cas dans le Miserere de Josquin). De fait, ce type de structure appelle plutôt la haute densité que le trio ou le quatuor ${ }^{86}$.

L'intégration du motto dans le contrepoint peut susciter un travail très subtil autour de l'imitation syntaxique. Il peut en effet entrer et sortir du jeu imitatif à tout moment, se laissant prendre dans la trame de l'exposition de chaque nouveau sujet, ce qui est le cas à l'incipit, lorsqu'il traite le même motif que les autres voix. Quand il y a deux textes, le cantus firmus tend à rester indépendant, comme maintenu en surplomb. Quand il n'y a qu'un seul texte dans la pièce, le compositeur peut aller jusqu'à créer un effet de refrain, les autres parties reprenant le texte du motif en même temps que la voce ostinata. C'est ce que fait Josquin dans son Miserere, qui forme d'ailleurs un cas un peu isolé. Un autre artifice consiste à placer une cadence mélodique à la fin de 1 'ostinato ${ }^{87}$; un jeu subtil de clausules plus ou moins polyphoniques s'engage alors.

Enfin, il est bon de suivre le traitement "harmonique" du cantus firmus à chaque intervention. Le compositeur prend-il soin de varier le contrepoint ou non? Cela a-t-il un sens? Le maintien du même cadre consonantique peut faire émerger le motif, tandis que la variété permet de le tenir caché. L'effet oratoire est diamétralement opposé et se lie parfois à une idée textuelle ou à un affect. Quand le cantus est transposé par degré, le problème se pose moins que lorsqu'il est immobile ou qu'il a seulement deux positions, ce qui crée un balancement entre deux pôles modaux. On verra plus loin comment Guerrero affirme sa subtilité en ce domaine et n'oublie pas de se préoccuper de tels paramètres, fondamentaux dans un type d'écriture comme celui-là.

-Compléments. Au chapitre III, le dernier paragraphe rassemble diverses remarques complémentaires, en particulier sur l'esthétique; la beauté des modèles guerrériens y est évoquée sans ambages. Il est suivi d'une conclusion.

86. L'exemple de Brumel (EX. 2) fait un peu figure d'exception. Son écriture très dépouillée pousse certainement le compositeur à limiter les dimensions de la pièce.

87. Voir T. L. de Victoria, Benedictus de la Missa de Beata Maria (cité plus haut, p. 57). 


\section{Analyse des onze pieces de F. Guerrero bâties sur un ostinato}

Avant de suivre le patron analytique précédent oeuvre par oeuvre et suivant l'ordre chronologique, il est bon de préciser un peu les limites de ce travail. Les dimensions de l'article imposent d'être bref et interdisent de consacrer plus d'une page à chaque pièce. La forme du discours se réduit donc à celle d'une fiche d'analyse, centrée sur la voce ostinata elle-même. Les onze paragraphes sont suivis de quelques remarques générales, pour conclure.

Dans chaque cas, on s'attache à suivre les filiations éventuelles de l'oeuvre, en particulier chez les autres compositeurs ibériques. Les originaux n'ayant pu être tous photographiés ${ }^{88}$, chaque ostinato est recopié, pour unifier la présentation. Le manque de place interdisant de fournir une transcription complète de l'ensemble, le lecteur est prié de se reporter à l'édition musicologique espagnole déjà citée. Enfin, les limites actuelles de cette dernière imposent le nombre de onze pièces. Les tomes suivants étofferont peut-être le corpus des motets comportant un ostinato, déjà remarquable. Ils sont attendus avec joie.

\section{Motet à 5 v. Veni Domine (1555), pour le temps de l'Avent}

-Texte: le motet est monotextuel; l'ostinato traite l'incipit Veni Domine et noli tardare. Il ne semble pas lié à un travail de solmisation. C'est une prière, presqu'une supplication -il dit ensuite: veni ad salvandum nos... ita consolaberis nos (viens nous sauver... ainsi tu nous consoleras). L'accent est mis sur la tristesse des hommes dans l'attente du Sauveur.

-Le cantus II chante l'ostinato. Etant toujours en surplomb, sauf au point d'orgue final, il est placé à découvert et s'entend particulièrement bien, ce que Guerrero a probablement calculé.

-Le motif, de style syllabique, comporte 11 notes, réparties sur 5 brèves. Il ne paraît pas emprunté au motet de Morales du même nom (1549) ni à une mélodie préexistante et n'apparaît pas non plus dans le motet Veni Domine de Las Infantas (1579). C'est une période fermée par une clausule de tenor.

-Disposition: la boucle motivique est double; le sujet est chanté deux fois, d'abord dans l'hexacorde naturel (avec abrégement de la valeur extrême), puis transposé à la 4te supérieure "par bémol" (Exemple 8), pour s'arrêter sur la finale modale. Il est à chaque fois couronné par un beau "fa sur hexacorde" très expressif. L'ensemble couvre 13 brèves (= le désarroi profond?) et se répète 3 fois. Il y a un long silence introductif de 7 brèves (= la grande peine?), réduit à 5 dès la première répétition. Les pauses initiales ont dès lors la même durée que les incises du sujet. Le total atteint 76 brèves $(=19 \times 4)$, nombre déjà relevé dans plusieurs œuvres du temps (Josquin, Agnus final de la Missa sixti toni, par exemple). En héraldique, le schéma du motif reconstitué s'appelle palé de 4 pièces; il est irrégulier et asymétrique. Le schéma de 1'ensemble est, lui, un vergeté de 16 pièces (Exemple 9).

88. Cela concerne les deux motets et la messe seculorum Amen. On représente schématiquement la voce ostinata dans chaque cas. 
Exemple 8: Motet Veni Domine (1555), cantus II (reconstitué).

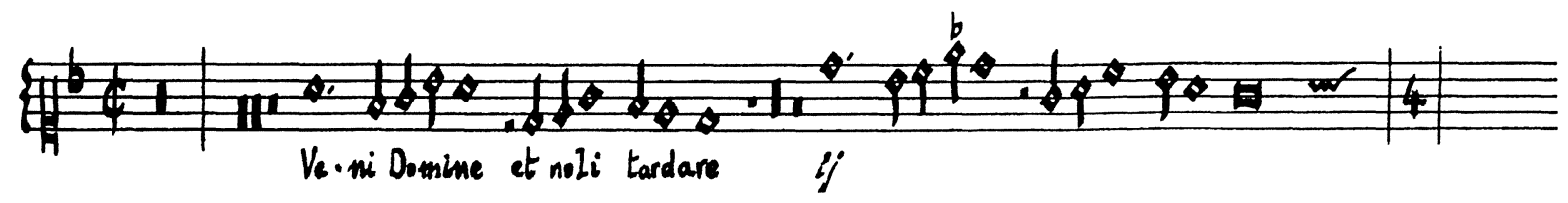

Exemple 9: Représentation globale de la partie vocale.

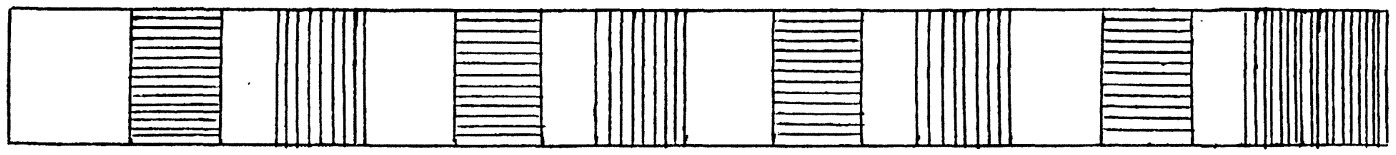

-La construction en imitation est remarquable. L'ostinato est toujours indépendant des autres parties, sauf au début et peu avant la fin, quand l'incipit du texte revient dans la polyphonie. Sa cadence de tenor reste isolée, en général. La mise en consonance est extrêmement variée.

-Remarques: Guerrero donne ici une magistrale leçon de composition, empreinte de modernité. L'ostinato couronne la polyphonie sans y entrer réellement et accroît le sentiment d'incertitude dans lequel baigne le texte. Sa plainte, qui se colore "d'harmonies" renouvelées, plane au dessus des autres parties, tristement serrées dans le grave. L'ostinato, non voilé, y prend un caractère litanique très prégnant. L'accent est donc mis sur l'art oratoire plutôt que sur la symbolique. Le choix esthétique place cette oeuvre dans le contexte de l'époque baroque naissante.

La filiation au motet de Morales (1549) est probable. Mais on relève de notables différences (motif à l'altus, caché au centre, structure à refrain, en deux parties distinctes, etc.). Guerrero a dû emprunter à son maître l'idée compositionnelle et quelques éléments de construction comme le nombre 5, qui sert d'étalon, mais il a mis davantage l'accent sur l'expression, semble-t-il. Quant au motet de Las Infantas, il marque de son originalité le troisième volet de cette trilogie ibérique.

\section{Missa Sancta et Immaculata à 5 v. (1566, $n^{\circ}$ I), Sanctus}

-Le texte est le Sanctus de la messe. L'ostinato répète "Sanctus, sanctus". C'est un appel, un "cri" de gloire.

-Le superius II assume la voce ostinata. Comme dans le précédent motet, il lance son appel au-dessus des autres parties jusqu'à la fin. La polyphonie est couronnée régulièrement par sa voix pleine de puissance et de solennité. L'ostinato agit ici par renforcement affectif, au-dessus d'un texte bref connu de tous, dont l'incipit résume à lui seul la teneur. 
-Le motif est emprunté au motet de Morales Sancta et immaculata (1545). C'est l'incipit de la pièce, confié au ténor (Exemple 10). Guerrero l'a modifié pour l'adapter au texte. Il comporte 6 notes, s'étend sur 5 brèves (en comptant la syncope initiale), cultive le style mélismatique et s'achève par une cadence phrygienne de tenor. Il a un caractère assez énergique avec son départ en syncope et ses valeurs, rapides pour un cantus firmus.

Exemple 10: 1) Morales;

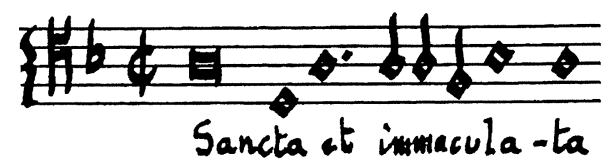

2) Guerrero. Source: Liber primus Missarum, BN, Res Vm a. 7.

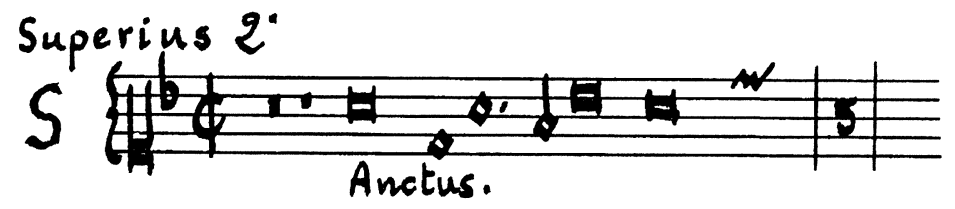

-La disposition de l'ostinato est assez claire. L'ensemble de 6 brèves unissant silence introductif ( 1 brève et demi) et motto ( 4,5 brèves) est chanté 5 fois, 3 fois "par nature" et deux fois à la 4te supérieure "par bémol". On note que la transposition est seulement indiquée par le signe de prise sur la cinquième ligne, peut-être pour éviter de compliquer la notation. La pièce comporte donc $5 \times 6=30$ brèves. On rencontre encore le nombre 5 , qui joue un grand rôle dans l'architecture guerrérienne, semble-t-il. En langage héraldique, la pièce représente un vergeté de 10 pièces.

Exemple 11: Sanctus de la Missa Sancta et immaculata, cantus II.

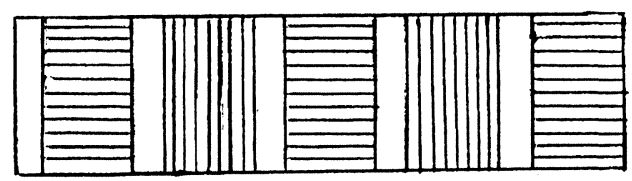

-Construction: le dispositif est très efficace, sur le plan rhétorique. L'appel glorieux des voix du ciel est très nettement perçu dans 1'aigu, au sommet d'une polyphonie assez dense et fervente. Le sujet se dégage nettement des autres parties vocales, au niveau du travail imitatif, et l'accompagnement en consonance est peu varié, renforçant la litanie de son soutien harmonique opiniâtre. 
-Remarque préalable: après cette messe, Guerrero n'a jamais plus utilisé l'ostinato deux fois de suite dans la même œuvre. Peut-être cela marque-t-il une évolution de son style, se dégageant peu à peu des usages franco-flamands et de l'influence de Morales.

-Le texte de l'ostinato dévoile l'origine de la parodie; il prononce 1'incipit du motet de Morales: Sancta et immaculata (1549). Cette apostrophe fervente accompagne le texte du Benedictus en formant une toile de fond, un Hintergrund marial plein de douceur. On admirera le tour de passe-passe du compositeur qui, après avoir traité une première fois l'incipit d'origine en ostinato sans son propre texte, le dévoile juste après dans le même groupe de pièces; mais il change de voix en cours de route.

-C'est ici le ténor qui chante le motif obstiné. Sa perception, dans 1'exécution vocale, est nettement moins aisée que précédemment, ce qui se conçoit bien dans le cadre d'un Benedictus, plus contemplatif. Donc, tout en dévoilant son modèle, Guerrero cherche à le cacher par la disposition polyphonique. Esthétiquement, on est proche de l'esprit du XVe s., qui cultive volontiers ce genre de tours. On se rapproche également des dispositions traditionnelles, accordant la primauté au ténor.

-Le motif utilise les mêmes notes que le Sanctus, mais reprend plus exactement l'incipit original. Il comporte 8 notes de style syllabique et s'étend sur 7 brèves, ou 9 en comptant le silence qui le précède (Exemple 12). Ecrit en valeurs lentes, il est plus solennel que dans le Sanctus, et mieux adapté au caractère du Benedictus.

Exemple 12: Missa Sancta et immaculata, Benedictus, ténor.

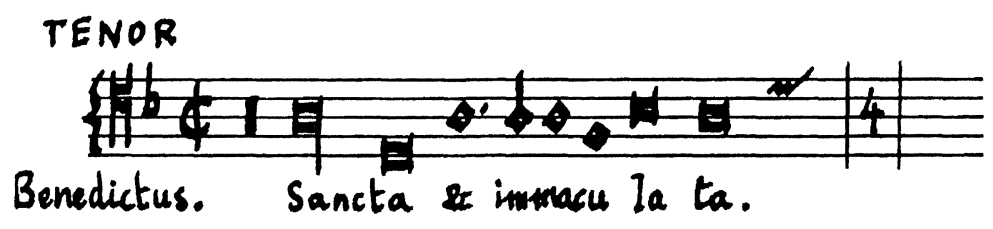

-La disposition suit le Sanctus précédent, avec seulement 3 reprises. On finit donc par la mélodie transposée et sur la finale intermédiaire D, mais cela n'a pas d'importance, car l'Hosanna revient au ton principal. L'architecture -un vergeté de 8 pièces- met en valeur des nombres très prisés comme le 7 (motif) et le $36(=4 \times 12$ brèves, durée totale de la pièce).

On note la brièveté des pauses intercalaires, tout comme dans le Sanctus. Le traitement de l'ostinato est plus proche de celui d'un cantus firmus, initerrompu, que celui d'un motto obstiné, tel qu'on le voit dans les motets; on en parle à nouveau dans la conclusion. 
Exemple 13: Schéma du Benedictus - messe Sancta et immaculata.

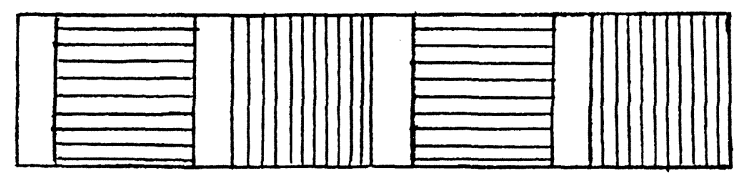

-La construction musico-syntaxique ressemble à celle du Sanctus.

-Remarques: c'est le seul Benedictus que Guerrero ait écrit de la sorte. C'est aussi le seul passage bitextuel de la messe. Comme par le passé, le Benedictus est le "coeur sensible" de l'oeuvre que le compositeur marque d'un trait de plume particulier.

4. Missa In te Domine à 4 v. (1566, $n^{\circ}$ II), Sanctus

-Le texte est celui du Sanctus, comme dans la messe précédente. L'appel est lancé trois fois de suite, suivant le canon de l'office.

-L'altus II, partie centrale du quintette, est choisi comme voix principale, écrite en ostinato. La disposition est assez habituelle, et apporte beaucoup de noblesse.

-Le motif, tout en valeurs longues, marqué par un ralentissement interne (les brèves s'allongent de moitié), contraste fortement avec le reste des voix, très volubiles. La brièveté des pauses intercalaires accentue l'aspect un peu archaïque de ce cantus firmus, traité en vox interna. La mélodie, d'origine inconnue, comprend 6 notes étendues sur 8 brèves ou 10, si l'on compte les silences qui précèdent. Elle est bâtie autour d'une double chute de 4tes qui lui donne un caractère campanaire. Peut-être était-elle jouée par des cloches.

Exemple 14: Sanctus de la messe In te Domine. Source: voir Exemple 10.

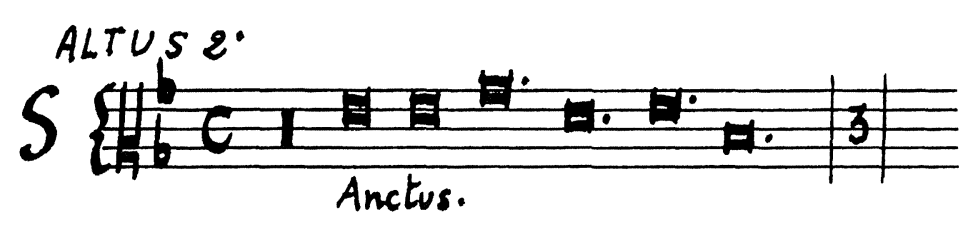

-La disposition de l'ostinato est fort simple. Elle forme un palé de 4 pièces long de 30 brèves, exactement comme le Sanctus de la messe précédente; du reste, la taille de ce type de pièces tourne souvent autour de 30-35 brèves. Le motif est chanté trois fois, sans modifications. Une telle rigueur convient à un Sanctus solennel, marqué traditionnellement par le nombre 3. Le mot latin est d'ailleurs entendu à l'altus II 9 fois $(=3 \times 3)$. 
-L’accompagnement en consonances est assez peu varié.

Exemple 15: Schéma du Sanctus - messe In te Domine.

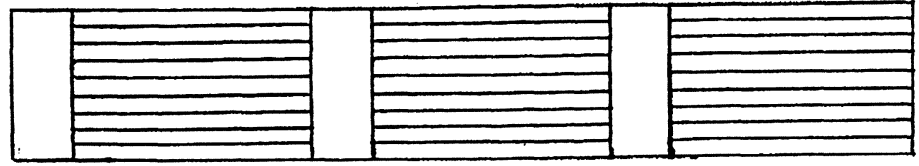

\section{Missa Congratulamini à 5 voix (1566, $n^{\circ}$ III), Agnus Dei I}

-Le texte est celui de l'office. La partie itérative répète seulement l'apostrophe Agnus Dei.

-Le cantus II chante le motif dans l'aigu, en se mêlant au premier superius. Tantôt en surplomb, tantôt proche de l'altus, il reste perceptible, sans pour autant dominer la polyphonie. Cette disposition est coutumière dans les œuvres de Morales. Elle prend ici un caractère symbolique: le choix d'une voix aigue correspond à l'évocation de Marie-Madeleine au tombeau, pleine de joie et de confiance.

-Le motif, de style syllabique, comporte 4 notes et recouvre 6 brèves. Ses 4 brèves pointées, déjà rencontrées dans la messe précédente, ont probablement un caractère symbolique: le 3, la divinité, et le 12, nombre parfait. Le dessin mélodique hexacordal (Exemple 16-2) est emprunté au motet Congratulamini de Guillaume Le Heurteur. C'est l'incipit du secundus altus (Exemple 16-1), adapté au texte et traité en augmentation.

Exemple 16: 1) motet Congratulamini (Le Heurteur), incipit de l'altus II.

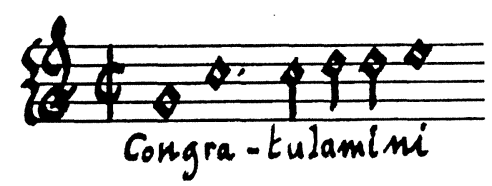

2) Guerrero, Agnus I de la messe Congratulamini (cantus II).

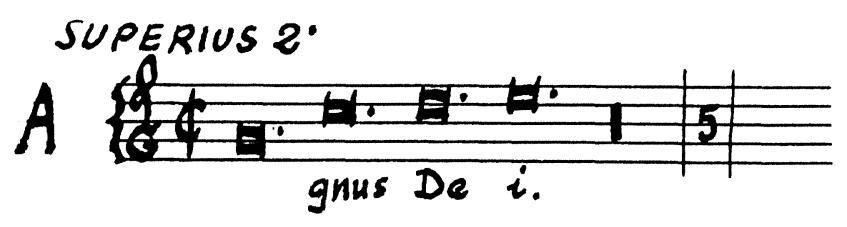

-La disposition de l'ostinato est très simple. Le sujet entre directement -ce qui est rareet se fait entendre tel quel 5 fois. On retrouve à nouveau ce fameux nombre 5 , "nombre de l'homme". La structure est de 4 pals blancs (Exemple 17) et le total atteint 38 brèves $(=19 \times 2)$. 
Les nombres règlent donc cette pièce d'une façon très stricte. Comme précédemment, Guerrero réduit les pauses intercalaires pour donner plus de présence au cantus firmus.

Exemple 17: Schéma de l'Agnus I - Missa Congratulamini.

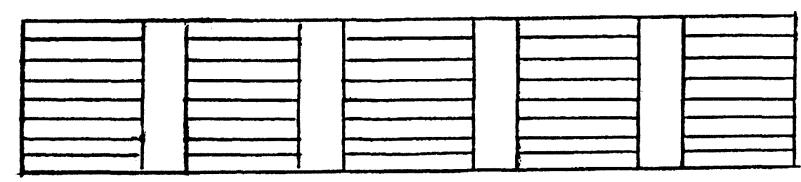

-La mise en contrepoint est remarquable de variété et de subtilité modale. Le contraste entre le cantus II et les autres voix, très volubiles et chantantes, est saisissant. Ici s'affirme le style guerrérien le plus pur, mêlant grâce et rigueur.

-Remarque: l'incipit de la pièce reprend l'original de Le Heurteur presqu'intégralement.

\section{Missa Beata mater à 4 v. (1566, $n^{\circ}$ VIII), Hosanna}

-La pièce est bitextuelle. L'ostinato répète l'incipit Beata mater, tandis que les autres voix énoncent le texte de l'office. Exceptionnellement, Guerrero choisit l'Hosanna ternaire pour placer une voce ostinata. Peut-être cela a-t-il un rapport avec le caractère alléluiatique du texte, apostrophe mariale pleine d'exaltation.

-L'altus chante le motif répétitif. C'est un choix très moralésien, qui s'inscrit bien dans la symbolique du texte; la voix d'altus peut fort bien représenter la vox matris (voix maternelle).

-Le motif de 5 notes, de style syllabique, couvre 7 brèves. Il est clos par une cadence de tenor sur d. Sa filiation avec l'antienne Beata mater n'apparaît pas clairement dans cette pièce. On note son aspect solennel, accentué par les valeurs lentes, le petit ambitus.

Exemple 18: Altus de la messe Beata mater.

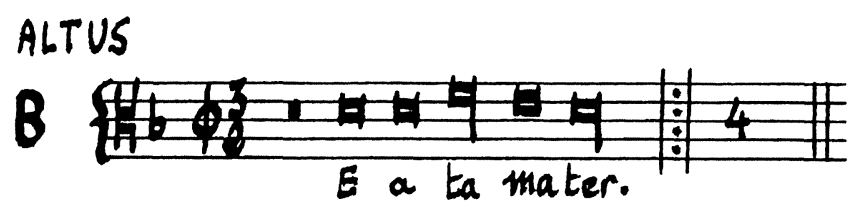

-La disposition de l'ostinato est simple. On entend le motif 4 fois sans changements, avec une seule pause brève intercalaire. Le cantus firmus est presque continu; cela gêne sa perception. Guerrero ne semble pas avoir souhaité le mettre particulièrement en valeur dans l'écriture. L'ensemble, de 32 brèves, forme un palé de 8 pièces. 
Exemple 19: Schéma de l'altus - Missa Beata mater, Hosanna.

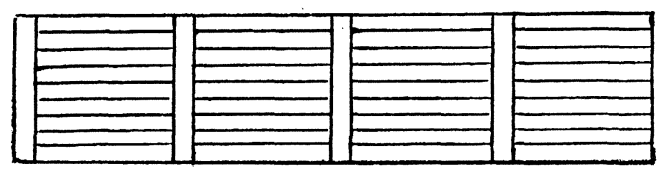

-La mise en contrepoint apporte peu de variété, conformément au caractère alléluiatique et litanique de la pièce. Il faut noter le grand contraste entre le cantus firmus axial et les autres voix, très volubiles.

-Une filiation possible conduit vers la messe Si bona suscepimus de Morales (1544), dont l'Hosanna présente un dispositif analogue.

\section{Motet Surge propera à 6 v. (1570)}

-Le motet est bitextuel, ce qui est rare chez Guerrero. Il s'agit certainement d'un hommage à ses prédecesseurs. L'ostinato lance 1'appel Veni sponsa Christi, incipit de 1'antienne du même nom, chantée aux Vêpres des Vierges. Les cinq autres voix traitent, elles, le texte du Cantique des Cantiques. Le lien entre les deux s'établit naturellement dans le contexte de cet office.

-Le cantus II est à nouveau la voix principale. Comme dans le premier motet analysé (Veni Domine), l'ostinato est en position dominante. Outre son aspect symbolique -il représente la voix féminine- son aspect rhétorique est remarquable. Sa situation lui donne le maximum d'efficacité. Il amplifie chaque vers du texte principal, en le placant sous le signe de la joie.

On rappelle à cette occasion que les motets mariaux ou dédiés à de saintes femmes présentent souvent un dispositif vocal analogue. Chez Guerrero, cela se manifeste dans Ave virgo sanctissima, Trahe me, etc.

-Le motif cite exactement 1'incipit de 1'antienne grégorienne. Quelques notes ont pu être déterminées, à l'origine, par la solmisation (les syllabes centrales “-ni spon-sa" correspondent à "mi-sol-la", lues par bécarre). Le sujet comporte 6 notes et couvre 10 brèves dans chaque partie du motet. Le nombre de pauses est variable. L'exemple suivant est une reconstitution.

Exemple 20: 1) motet Surge propera, première partie, cantus II.

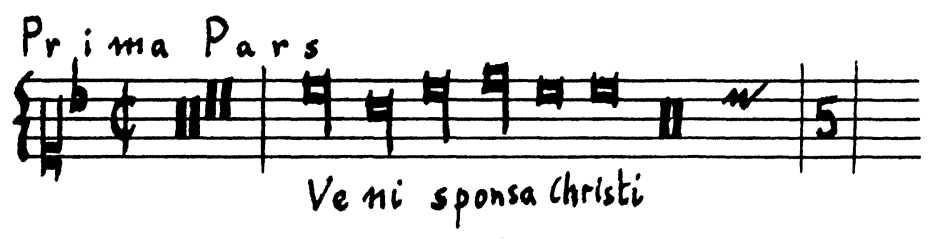


2) seconde partie.

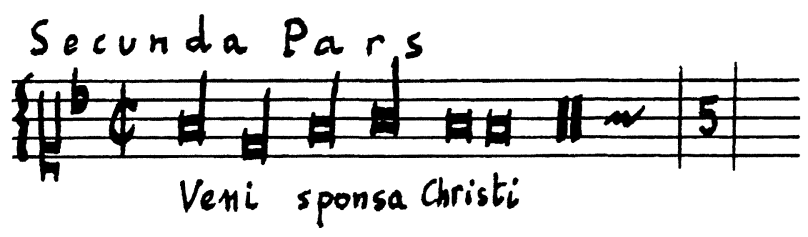

-La disposition de l'ostinato est très complexe. C'est le seul qui utilise la transposition par degré, en hommage à la tradition josquinienne. De plus, la forme varie d'un volet de l'oeuvre à l'autre. Dans la première partie, un long silence introductif ( 8 brèves) précède les 5 interventions du motif, séparées par 4 brèves intercalaires. L'ensemble atteint le fameux nombre $76(=19 \times 4)$, point d'orgue compris. Le motif parcours le pentacorde cc-f, pour rejoindre la finale (f) du 6e mode. L'aspect général est un vergeté de 10 pièces, adextré. La seconde partie opère le mouvement inverse, remontée symbolique vers la lumière céleste. Le schéma est plus ramassé, sans pause de départ, et n'embrasse que 66 brèves; au total, l'oeuvre compte 142 brèves, total proche de $144=12 \times 12$. L'héraldiste compte 4 pals blancs.

Exemple 21: 1) motet Surge propera, schéma de la première partie.

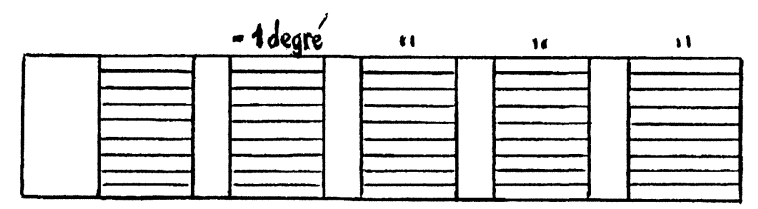

2) schéma de la seconde partie.

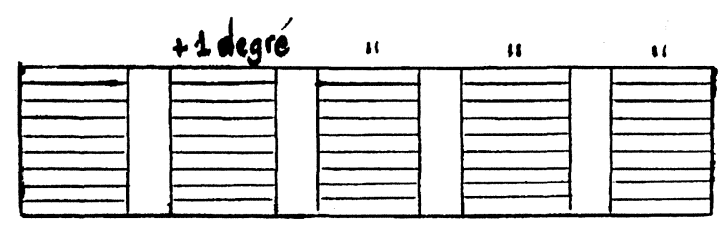

-La construction syntaxique est remarquable. L'ostinato s'immisce harmonieusement dans (presque) toutes les périodes du texte. Il exerce une fonction de soulignement, d'accompagnement qui tranche avec celle que Guerrero lui attribue dans les messes. Le motet favorise, il est vrai, la précision et l'importance du travail contrapuntique, du moins chez ce compositeur. Quant à la variété texturale et "harmonique", elle est richement mise en jeu.

-La filiation avec le Miserere de Josquin s'établit au niveau technique. Mais, dans la production du XVIe s., on ne trouve guère de pièces analogues traitant les mêmes textes. Pour citer un exemple, le motet Tota pulchra es de Clemens non Papa (1554) mêle le Cantiques des 
Cantiques à une autre invocation dédiée à une sainte: Sancta Margaretha ora pro nobis (Sainte Marguerite, prie pour nous). On relève, en revanche, quelques similitudes musicales entre le motet de Guerrero et un motet donné par H. Finck (op. cit. 1556, f ${ }^{\circ}$ Hhij), bâti à 4 voix sur le même ostinato, et aussi avec le Tu es Petrus (1546) de Morales.

-Remarque: l'architecture met en valeur les nombres 5 et 10 , déjà rencontrés.

8. Missa Ecce sacerdos à 5 v. (1582), Kyrie II

-La pièce est bitextuelle. L'ostinato accompagne le Kyrie avec les paroles Ecce sacerdos magnus (Voici le grand prêtre), qui sonnent comme une devise annonçant l'arrivée d'un haut personnage. En réalisant cette superposition de textes dès la première partie de la messe, Guerrero dévoile d'un facon inhabituelle l'origine musicale de son oeuvre.

-L'altus I chante l'ostinato, en restant caché au centre de la polyphonie. Le Maître a confié ce "cri" à une voix centrale, probablement masculine, symbolisant peut-être une sorte de héraut.

-Le motif: c'est l'incipit de l'antienne des Vêpres d'un confesseur. Il rassemble 7 brèves de style syllabique. Il martèle plusieurs fois le f en valeurs égales et prend de ce fait un caractère martial.

-La disposition de 1'ostinato combine deux procédés de variation, mélodique et rythmique. Guerrero lie en effet transposition hexacordale et diminution du tactus, employée ici pour la première fois. La pièce est ainsi divisée en deux parties inégales. Le sujet est d'abord énoncé à deux hauteurs différentes en temps imparfait diminué, avec une pause longue intercalaire, puis le tout est repris en double-diminution, suivant le signe de mensuration. La notation d'origine autorise une compréhension très aisée du phénomène (Exemple 22). Le tout rassemble 27 brèves plus une en point d'orgue, soit $7+7$ brèves +4 pauses $=18$ brèves +9 brèves $+1=28$, soit $4 \times 7$. Le nombre 7 , nombre parfait, exerce un rôle actif dans cette construction. Le schéma général met en valeur un palé de 8 pièces en parti senestré (= dissymétrique, plus développé à gauche).

Exemple 22: Messe Ecce sacerdos, Altus I (Kyrie II). Source: Missarum Liber 2 (1582), BN, Res F872.

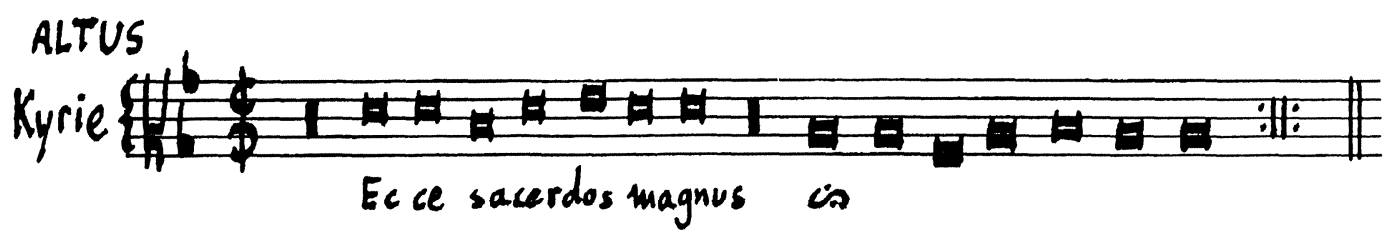


Exemple 23: Schéma de la partie vocale ci-dessus.

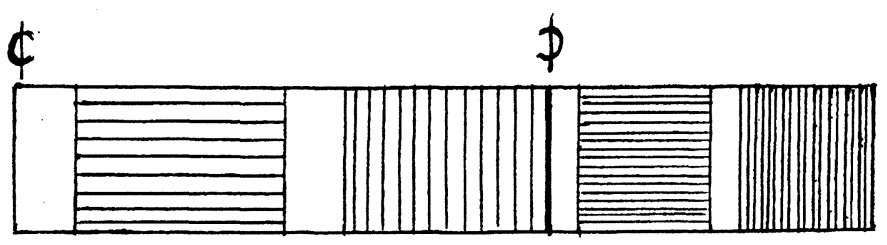

-La construction syntaxique est la même que dans les autres fragments de messe guerrériens. La seule particularité est l'accélération subite du cantus firmus au sein de la polyphonie. "L'harmonie" est marquée par une belle variété. On notera que l'ostinato ne termine pas sur la finale modale.

-Les filiations de l'oeuvre conduisent vers Josquin des Prez (par exemple le motet Illibata), mais aussi vers Morales, dont Guerrero a pu s'inspirer. Le Kyrie II de la messe Ave Maria de Cristobal (1544) exploite le même procédé. La diminution est d'ailleurs traditionnellement appliquée à cette partie de la messe. C'est le seul Kyrie traité de la sorte par Guerrero.

\section{Missa della Batalla à 5 v. (1582), Agnus I}

-Le texte est celui de l'office. L'ostinato répète l'apostrophe Agnus Dei, amplifiant la prière faite à l'Agneau.

-Le cantus II est la voce ostinata. Il évolue dans le medium aigu et s'entend à découvert lorsqu'il attaque les cc aigus. Sa présence est un peu discrète, mais bien perceptible. Cette disposition, on l'a dit plus haut, doit beaucoup à l'influence de Morales.

-Le motif est 1'incipit Escoutez de la chanson La Guerre de Janequin, mainte fois parodiée au XVIe s. L'adaptation au texte a réduit la mélodie à un simple squelette de 4 notes, traitées en style syllabique. Ce sujet s'étend sur 6 brèves.

Exemple 24: Missa della Batalla, Agnus I, cantus II. Source: voir Exemple 22.

\section{CANTUS II}

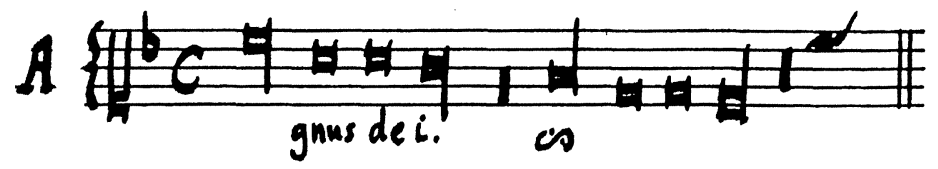

-La disposition de l'ostinato n'est pas nouvelle. Le sujet intervient à deux hauteurs différentes: par bémol et en musica ficta, par transposition sur Bmol grave. Il finit d'ailleurs sur cette position, à la 5te de la finale modale, comme aux paragraphes 3,8 et 11 de ce chapitre. Il y a deux répétitions intégrales, oubliées par l'éditeur. L'ensemble couvre 47 brèves. On notera le 
dessin pentatonique formé par le motif et sa transposition. Le schéma forme 5 pals blancs, en l'absence de silence introductif.

Exemple 25: Représentation du cantus II ci-dessus.

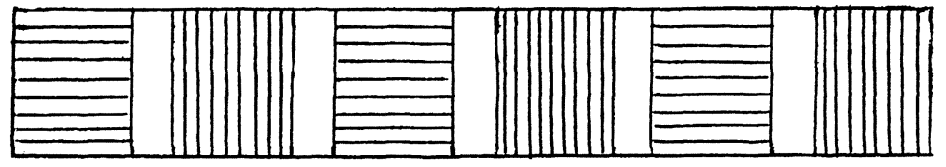

-La mise en contrepoint témoigne d'un grand souci de variété. Le cantus firmus est, comme à 1'accoutumée, presque continu et indépendant du travail imitatif. Cependant, Guerrero a eu l'excellente idée de le faire entrer en canon avec le cantus I pour commencer, ce qui donne plus de corps au sujet.

-Remarque: il est difficile de relier cette pièce à une autre, tout au plus y a-t-il quelque similitude avec le Sanctus de la Missa l'Homne armé à 5 v. de Morales.

10. Missa Simile est à 4 v. (1582), Agnus I

-Le texte est celui de l'office. Dans l'édition originale (Roma, 1582), l'ostinato dit l'incipit Agnus Dei. La partition moderne en décide autrement. On préfère se ranger ici à l'indication d'époque. C'est la troisième fois que Guerrero traite ainsi l'Agnus. Cela convient à l'aspect litanique et implorant de cette partie.

-Le ténor répète un motif de 7 notes d'origine inconnue. Ainsi placé, le motto est peu perceptible. Le compositeur inscrit la pièce dans la tradition du cantus firmus tenorizans, très solennel.

-La disposition de l'ostinato est nouvelle, dans l'oeuvre du Maître. Elle porte une règle explicative ou "canon", qui propose le déchiffrage d'une énigme: vado et venio ad vos (je vais et je viens vers vous; d'après Jean 14, 28). Chaque boucle motivique est donc double, et fait entendre le motif par mouvement droit et par mouvement rétrograde. Cela représente 16 brèves, que le compositeur reprend une fois. Le total atteint donc 32 brèves, portées à 36 par le point d'orgue, qui inscrit la pièce dans un nombre très représentatif $(36=12 \times 3)$. Le schéma donne un palé de 8 pièces en parti senestré (= asymétrique, plus développé sur la gauche).

Exemple 26: Missa simile est, ténor de l'Agnus I.

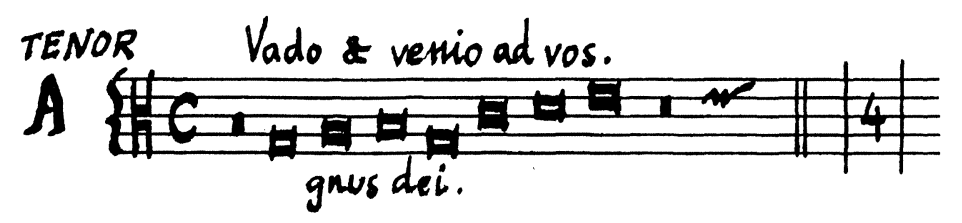


Exemple 27: Schéma du ténor précédent. Le quadrillage indique la rétrogradation du motif.

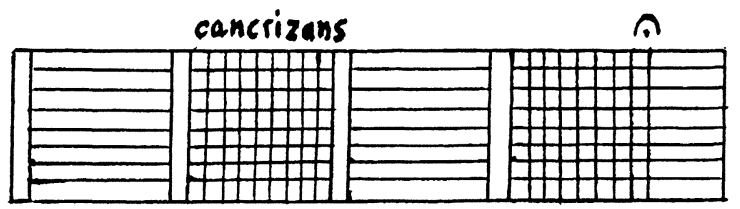

-Le ténor a l'allure d'un cantus firmus. Le mouvement rétrograde de la mélodie amplifie cet aspect, en interdisant toute reconnaissance auditive. On note au passage combien l'interprétation symbolique de ce motus cancrizans est délicate. Il faut remarquer également la beauté de la mise en consonances.

\section{Missa seculorun Amen à 4 v. (1597), Agnus I}

-Le texte est celui de la messe. Dans 1'édition moderne -la seule source disponiblel'ostinato traite également ce texte en le marquant d'une ellipse. Ceci serait certainement digne d'une discussion musicologique, malheureusement peu à sa place dans cet article.

-Le ténor est la partie principale, comme dans 1'Agnus précédent. Il a le même caractère de cantus firmus.

-Le motif de 6 longues est bâti sur une terminaison psalmodique du VIIIe ton. Il est clos par une cadence de tenor sur G.

-La disposition de l'ostinato rejoint celle du paragraphe 8 (Missa Ecce sacerdos). La boucle motivique fait entendre le sujet à deux hauteurs différentes: par bécarre puis par nature. Le ténor se termine d'ailleurs sur la 5 te supérieure de la finale. On compte 28 brèves, qui sont ensuite reprises en diminution. A cet effet, la partie vocale doit certainement porter un signe de mensuration adéquat, comme dans le $\mathrm{n}^{\circ} 8$; on le note dans 1 'exemple suivant. Le schéma est un 3 pals blancs en parti senestré (= dissymétrique, plus développé à gauche) de 41 brèves.

Exemple 28: Missa seculorum Amen, ténor de l'Agnus I, reconstitué.

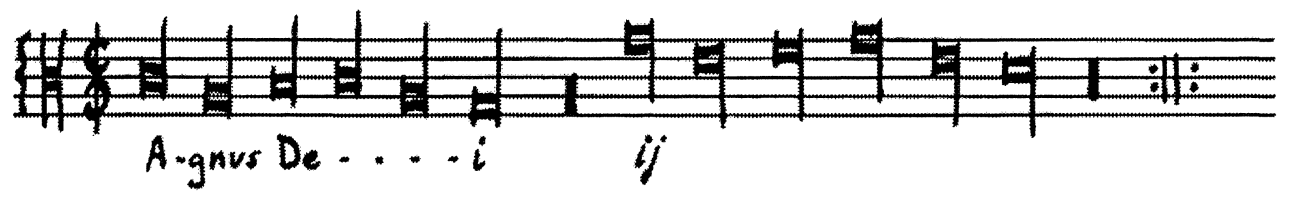


Exemple 29: Schéma de ténor précédent.

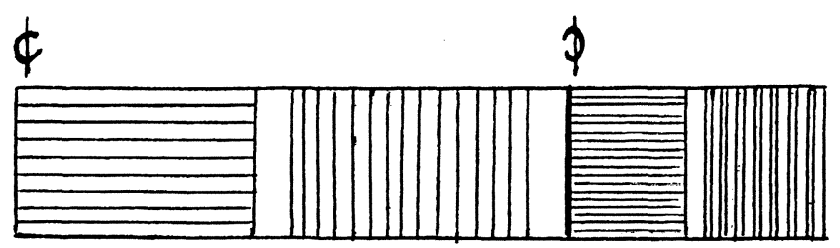

-Remarquer la variété des consonances et la souplesse des lignes mélodiques fleuries.

\section{Conclusion}

Cette courte partie analytique montre comment le respect de la tradition franco-flamande et espagnole marque l'oeuvre de Francisco Guerrero. Se pliant encore fréquemment aux exigences du travail sur ostinato, réputé contraignant et difficile, il rend hommage à ses illustres prédécesseurs, Josquin en tête, et à son maître Cristobal de Morales. Sans innover dans ce domaine, il exploite néanmoins la plupart des dispositifs du genre, avec transpositions et proportions rythmiques, et réussit à se renouveler presque constamment. Il traite l'écriture avec une certaine simplicité, suivant le goût de son époque, où l'heure n'est plus tout à fait à la complexité mathématique. Il y met plutôt l'élégance suprême qui caractérise sa manière, et cultive la beauté des consonances, la souplesse des lignes mélodiques et la suavité des parcours modaux.

Comme les compositeurs de son temps, il accorde une importance particulière -semble-til- à 1 'architecture et aux nombres qui la règlent ${ }^{89}$, tout en ménageant quelques interprétations symboliques bien ancrées dans la culture renaissante. A ce propos, on remarque l'omniprésence des nombres 5 (comme chez Morales), 6 et 7. On note aussi l'importance accordée à la place de l'ostinato; les voix élevées y représentent souvent l'élément féminin ou virginal.

S'estimant certainement plus libre dans le domaine du motet que dans la messe, Guerrero nous livre là des chefs-d'oeuvre qui transcendent la technique de l'ostinato. Un texte comme Veni Domine, déjà magnifié par Morales, trouve son expression la plus intense sous la plume du Sévillan. Il sait en effet donner à la disposition musicale une grande efficacité oratoire, en plaçant, par exemple, la voce ostinata dans la partie qui domine la polyphonie. C'est l'aveu d'un

89. Rolf Dammann (op. cit. 1953, p. 17) cite à ce propos des témoignages éloquents, en particulier un extrait de la Bible (Liber Sapientiae Salomonis, 11,21) qui, selon lui, aurait servi de précepte architectural pour tous les arts; cet aphorisme commande de tenir compte du nombre constructeur, en ces termes: omnia et numero et pondere disposuisti, /Deus/. (/Dieu/, tu as réglé toutes choses avec nombre, poids et mesure). Cette phrase revient, un peu déformée, dans la Préface du Speculum musicae de Jacobus von Luttich (circa 1323): Deus omnia fecit in numero, pondere et mensura (Dieu a tout créé avec nombre, poids et mesure). C'est ce que Dammann relie au Prinzip der Zahl (principe du nombre). 
réel souci rhétorique, cultivant la convenance du discours musical, bien naturel chez un compositeur de la fin du XVIe s. En effet, après le concile de Trente, il est d'usage de chercher à faire vivre davantage le texte et de se mettre à son service par tout moyen utile, en prenant modèle sur les arts de l'éloquence ${ }^{90}$. Guerrero est réputé pour cela, si l'on en croit le célèbre témoignage de Cristoval Mosquera de Figueroa91: "entre los Españoles con justo titulo se señala Francisco Guerrero... el qual fue de los primeros, que en nuestra naçion dieron en concordar con la musica el rithmo, y el espiritu de la poesia... aplicando al bivo con las figuras del canto la mesma significacion de la letra" (parmi les / compositeurs / espagnols, Francisco Guerrero se fait remarquer à juste titre, lui qui fut parmi les premiers dans notre nation à mettre en accord la musique avec le rythme et l'esprit de la poésie... en appliquant la signification-même du texte avec la meilleure convenance ${ }^{92}$, grâce aux figures du chant).

Dans la messe, au contraire, le Maître s'en tient à des modèles plus classiques, hérités en particulier de Morales. L'ostinato est traité sous forme de cantus firmus, brièvement interrompu par de courtes pauses. Il se cache plus volontiers au coeur de la polyphonie, suivant l'ancienne règle. Il intervient généralement dans l'Agnus, où son caractère s'accorde bien avec la prière, de forme litanique. On le rencontre une fois seulement dans l'Hosanna, le Kyrie et le Benedictus. Dans bien des cas, la symbolique permet d'envisager l'origine de ce choix. Les pièces les plus traditionnelles -Sanctus, Agnus sur ostinato- présentent souvent une disposition-modèle où l'aspect technique semble prendre le pas sur l'expression pure. Il est bon de lire à ce sujet le Sanctus de la messe In te Domine.

De même, les dispositions bitextuelles, honorées par Guerrero, paraissent un peu surannées à la fin du XVIe s. Là s'affirme le goût tout ibérique pour la tradition la plus haute, attachement qui se perpétue jusqu'au milieu du siècle suivant. Mais qu'importe, confronté à des impératifs culturels très prégnants dans l'Espagne de Philippe II, Francisco Guerrero garde la plus grande sérénité, conforté par sa maîtrise transcendante et sa renommée internationale, pour nous laisser des exemples prestigieux usant des dispositifs les plus coercitifs. Cette partie de sa production, peut-être la plus sophistiquée, baigne dans la lumière de son génie.

90. Voir à ce sujet Joël Heuillon: Introduction aux Préfaces des Nuove Musiche de Giulio Caccini, Paris, Cahiers GKC, La Musique éloquente, vol. 2, 1993.

91. Prologue de 1'édition des Canciones y Villanescas espirituales, Venezia, Vincenti, 1589, proposé par Miguel Querol Gavalda, in: Opera omnia, vol. I, Barcelona, 1982, p. 12.

92. Au sens cicéronien du terme, c'est-à-dire adéquation, efficacité oratoire. 